\title{
Three-Dimensional Alignment and Corotation of Weak, TC-like Vortices via Linear Vortex Rossby Waves
}

\author{
Paul D. Reasor and Michael T. Montgomery \\ Department of Atmospheric Science, Colorado State University, Fort Collins, Colorado
}

(Manuscript received 20 March 2000, in final form 26 January 2001)

\begin{abstract}
The vertical alignment of an initially tilted geostrophic vortex is shown here to be captured by linear vortex Rossby wave dynamics when the vortex cores at upper and lower levels overlap. The vortex beta Rossby number, defined as the ratio of nonlinear advection in the potential vorticity equation to linear radial advection, is less than unity in this case. A useful means of characterizing a tilted vortex flow in this parameter regime is through a wave-mean flow decomposition. From this perspective the alignment mechanism is elucidated using a quasigeostrophic model in both its complete and linear equivalent barotropic forms. Attention is focused on basicstate vortices with continuous and monotonically decreasing potential vorticity profiles.

For internal Rossby deformation radii larger than the horizontal scale of the tilted vortex an azimuthal wavenumber 1 quasi mode exists. The quasi mode is characterized by its steady cyclonic propagation, long lifetime, and resistance to differential rotation, behaving much like a discrete vortex Rossby wave. The quasi mode traps disturbance energy causing the vortex to precess, or corotate, and thus prevents alignment. For internal deformation radii smaller than the horizontal vortex scale, the quasi mode disappears into the continuous spectrum of vortex Rossby waves. Alignment then proceeds through the irreversible redistribution of potential vorticity by the sheared vortex Rossby waves. Further decreases in the internal deformation radius result in a decreased dependence of vortex evolution on initial tilt magnitude, consistent with a reduction of the vortex beta Rossby number.

These results are believed to have relevance to the problem of tropical cyclone (TC) genesis. Cyclogenesis initiated through the merger and alignment of low-level convectively generated positive potential vorticity within a weak incipient vortex is captured by quasi-linear dynamics. A potential dynamical barrier to TC development in which the quasi mode frustrates vertical alignment can be identified using the linear alignment theory in this case.
\end{abstract}

\section{Introduction}

A vertically tilted vortex in the atmosphere either resists external forcings to align or succumbs to such influences by irreversibly shearing apart. The question of how and under what circumstances vertical alignment occurs has been addressed in previous studies with large-scale atmospheric and oceanic circulations in mind (McWilliams 1989; Polvani 1991; Viera 1995; Dritschel and de la Torre Juárez 1996; Sutyrin et al. 1998). The conceptual picture put forth for the evolution of an unforced tilted vortex is that potential vorticity (PV) at upper levels is advected by the vertically penetrating flow associated with the PV at lower levels, and vice versa. In the large-tilt parameter regime emphasized in prior studies the mutual advection and subsequent vortex evolution were found to depend on the magnitude of the initial vortex tilt. Therefore, to predict the evo-

Corresponding author address: Dr. Paul D. Reasor, NOAA/AOML Hurricane Research Division, 4301 Rickenbacker Causeway, Miami, FL 33149.

E-mail: reasor@aoml.noaa.gov lution of a tilted vortex, solutions to the nonlinear equations of motion were sought. Polvani (1991) showed that the evolution of a tilted vortex patch in a two-layer quasigeostrophic (QG) model can be predicted on the basis of its nearness to a geometric configuration known as a stable V state (vortex state) in which the PV precesses without change of shape at a constant rate. The tilted $\mathrm{V}$ state is an exact solution to the nonlinear equations of motion. A tilted vortex will precess when near a V state and tend to align when initially far from any $\mathrm{V}$ state. The vortex in the latter case approaches a circular barotropic configuration through filamentation and axisymmetrization (e.g., Melander et al. 1987).

Here a new and complimentary approach to understanding the unforced vertical alignment process for continuously distributed vortices is developed utilizing the fact that for overlapping upper- and lower-level PV cores, the vortex is meaningfully decomposed into an azimuthal mean and departure therefrom (i.e., a wavemean flow partitioning). The analysis is restricted to vortices with broad vorticity distributions, consistent with observations of weak tropical cyclones (TCs) (e.g., 
Azimuthal Mean Tangential Wind
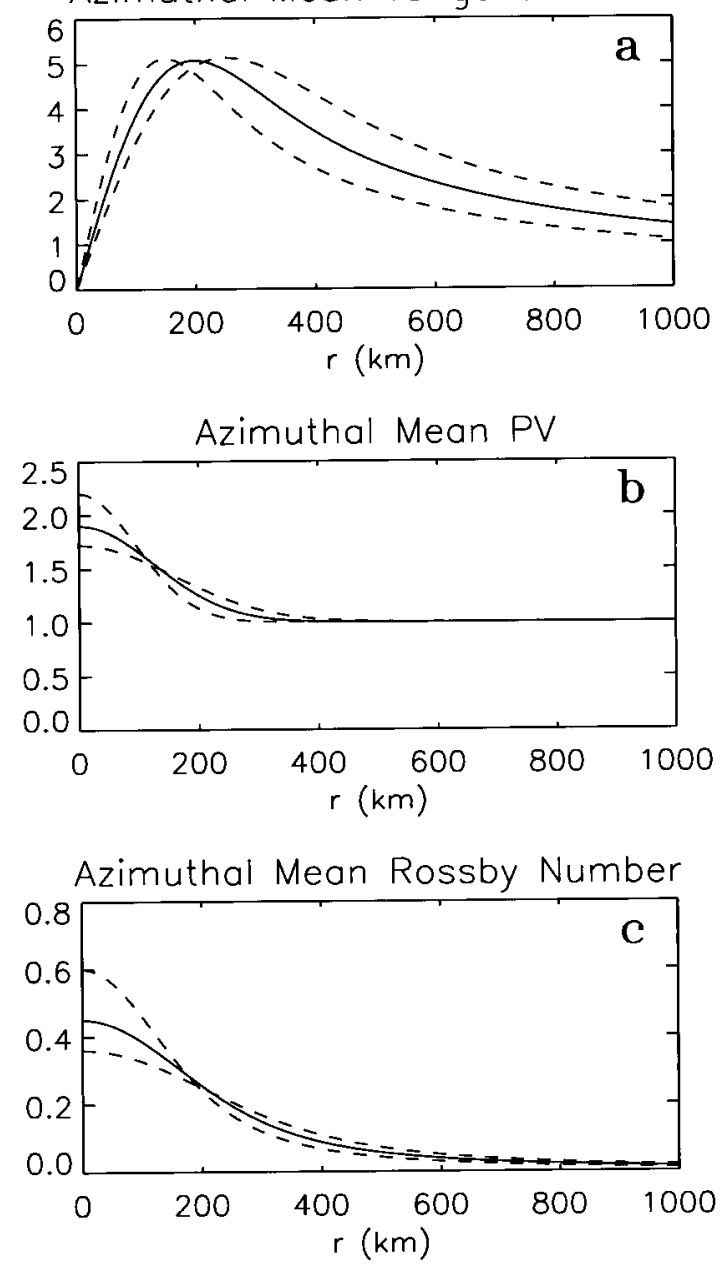

FIG. 1. The azimuthal-mean vortex (solid) used in all simulations unless stated otherwise in the text. The vortices depicted by the dashed curves are described in appendix B. The (a) tangential wind is in units of $\mathrm{m} \mathrm{s}^{-1}$ and the (b) PV is in units of $10^{-4} \mathrm{~s}^{-1}$. The (c) Rossby number is defined as $\bar{\Omega} / f$, where $\bar{\Omega}$ is the azimuthal-mean angular velocity.

Willoughby 1990). We explicitly simulate the linear interaction of perturbation and mean flow as well as the fully nonlinear vortex evolution to elucidate the alignment dynamics. Vortex Rossby waves (the vortex analogue of planetary Rossby waves, but with the azimuthal-mean vorticity gradient serving as the restoring mechanism and the differential rotation of the vortex tending to shear the waves) are shown to play a key role in the vertical alignment process, just as they do in the two-dimensional and quasi-two-dimensional vortex axisymmetrization process described by Montgomery and Kallenbach (1997, hereafter MK) and later confirmed by Montgomery and Enagonio (1998, hereafter ME98), Möller and Montgomery (1999, 2000) and Enagonio and Montgomery (2001). A simple conceptual picture of vortex alignment emerges for small vertical tilts based solely on linear dynamics. Of course linear dynamics can only be expected to capture alignment for a limited range of vortex tilts, but it will be shown that this range can be surprisingly large. A physical explanation is provided for why linear thinking can be applied to vortices exhibiting large initial tilts in certain parameter regimes.

Recently, Dritschel (1998) demonstrated how vortex axisymmetrization in inviscid two-dimensional flow can be inhibited for sufficiently steep-edged vortices. He argued that in the two-dimensional relaxation of an initially random distribution of vorticity on an $f$-plane, steep-edged vortices are more likely to form than broadly distributed vortices, and therefore serve as a more appropriate starting point for addressing the axisymmetrization problem. If this argument is extended to tilted vortices in three dimensions, there is the suggestion that the basic mechanics of vortex vertical alignment will be altered in going from broad to steep-edged vortices. We mention this possibility as a point of interest, but, as discussed above, focus our attention on the broad profiles commonly observed in developing TCs.

Recent dynamical studies of TCs have emphasized how such vortices resist the effects of vertical shear and other external strains during all life cycle stages (Jones 1995; Smith et al. 2000). Jones (1995) considered the evolution of a hurricane-like vortex embedded in a vertically sheared zonal flow. Her physical interpretation of the evolution from aligned to tilted vortex largely follows that of Polvani (1991) and other similar studies. As a prototype for the TC problem in vertical shear, Smith et al. (2000) proposed a two-layer nonlinear analogue model, solvable analytically. Extending Polvani (1991), they presented a portrait of upper- and lowerlevel vortex trajectories as a function of shear magnitude, vortex strength, and coupling between layers. The qualitative results of the model were verified using a two-layer QG model, but it should be noted that their analogue model becomes singular as the initial upperand lower-level PV separation goes to zero. Their analogue model also prohibits deformation of the vortex core, which therefore precludes vertical alignment in the unforced problem. Our work compliments these studies by taking a step back and exploring the unforced problem for small but finite-amplitude initial tilts. According to our interpretation of the unforced vortex dynamics, the addition of vertical shear simply makes the problem a forced linear one as long as departures from vertical alignment are not too great. In the context of TCs, observations suggest that the small-tilt limit is the relevant parameter regime (e.g., Marks et al. 1992; Reasor et al. 2000). The extension of the current work to the forced problem will be considered in a forthcoming publication.

Although the alignment dynamics of mature hurricanes is of great interest, here we consider the simpler problem of QG vortex alignment and its application to 
TC genesis as a first step. An axisymmetric view of TC genesis has been offered by Bister and Emanuel (1997) based on observations of TC Guillermo (1991) and supporting axisymmetric numerical simulations. A reanalysis of the Guillermo Doppler wind data by Bracken (1999), however, suggests a more prominent role played by asymmetries during the initial stages of genesis. ME98 presented a nonaxisymmetric model for genesis in which a preexisting midlevel vortex merges with nearby convectively generated low-level positive PV. Through this asymmetric process a cyclonic circulation is established beneath the midlevel vortex and a warm core is developed. This merger of PV within the lower to middle troposphere is similar to the alignment of a tilted vortex.

The paper is therefore organized as follows. In section 2 the three-dimensional and truncated equivalent barotropic QG models are described. The evolution of an initially tilted vortex is examined in section 3 . In section 4 we apply the linear alignment ideas to the problem of TC genesis via the three-dimensional merger of an isolated PV anomaly within a preexisting vortex. As the vortex becomes more vertically coherent in the lower to middle troposphere, the tilted vortex ideas of section 3 and their extension to finite Rossby numbers then become more directly applicable. In section 5 we present conclusions and indicate directions for our future research.

\section{Model descriptions}

\section{a. Three-dimensional QG model}

Motivated by the geostrophic nature of large-scale flows in the middle-latitude atmosphere and ocean, the QG system has been used extensively in the study of vortex merger and alignment (McWilliams 1989; Polvani 1991; Viera 1995; Dritschel and de la Torre Juárez 1996; Sutyrin et al. 1998). Its utility as a benchmark model for testing basic dynamical theories also has been exploited in recent studies of TCs (ME98; Smith et al. 2000). The PV conservation equation and invertibility relation on an $f$ plane in pseudo-height coordinates (Hoskins and Bretherton 1972) are, respectively,

TABLE 1. Parameters used in the control simulations. For the benchmark basic-state vortex the two parameters that define each simulation are the inverse internal Rossby deformation radius $\left(\gamma_{1}\right)$ and tilt amplitude $(\alpha)$. Useful parameters derived from these two are the upperand lower-level PV intercentroid separation distance $\left(d_{i}\right)$ and vortex beta Rossby number $\left(R_{\beta}\right)$. The latter is defined in section $3 \mathrm{~b}$.

\begin{tabular}{cccc}
\hline \hline$\gamma_{1}\left(\times 10^{-6} \mathrm{~m}^{-1}\right)$ & $\alpha(\mathrm{km})$ & $d_{i}(\mathrm{~km})$ & $R_{\beta}(\mathrm{km})$ \\
\hline 3.14 & 0.1 & 41 & 0.08 \\
3.14 & 0.3 & 123 & 0.25 \\
3.14 & 0.5 & 175 & 0.50 \\
20.0 & 0.3 & 123 & 0.08 \\
\hline
\end{tabular}

$$
\begin{aligned}
\frac{\partial q}{\partial t}+J(\psi, q) & =0, \\
q & =f_{0}+\nabla_{h}^{2} \psi+\frac{1}{\rho_{0}} \frac{\partial}{\partial z}\left(\frac{\rho_{0} f_{0}^{2}}{N^{2}} \frac{\partial \psi}{\partial z}\right),
\end{aligned}
$$

where $q$ is the PV, $\psi$ the flow streamfunction, $\nabla_{h}^{2}$ the horizontal Laplacian, $\rho_{0}$ the pseudodensity, $f_{0}$ the constant Coriolis parameter, and $N^{2}$ the static stability. In Cartesian coordinates the Jacobian, $J(\psi, q)=\partial \psi / \partial x$. $\partial q / \partial y-\partial \psi / \partial y \cdot \partial q / \partial x$. The details of the three-dimensional numerical model (henceforth referred to as QG3D) are described by ME98. One difference from ME98 is that in the nondimensional numerical simulation of (1)-(2) we will not require the horizontal scale, $L$, equal the Rossby deformation radius, $L_{R}=N H / f_{0}$, where $H$ is the model depth. The thermal vorticity in the nondimensional invertibility relation (ME98, Eq. 5) is then multiplied by the vortex Burger number, $\left(L_{R} /\right.$ $L)^{2}$. Unless otherwise stated, no explicit diffusion is included in the QG3D model.

\section{b. Equivalent barotropic $Q G$ model}

A tilted PV column vertically bounded by rigid lids can be decomposed into a barotropic mode and internal baroclinic modes. In general the interior flow results from both interior PV and potential temperature anomalies on the horizontal boundaries (Hoskins et al. 1985). Hurricane observations show that the potential temperature gradients on the horizontal boundaries are weak (e.g., Hawkins and Rubsam 1968). Consistent with these observations and recent theoretical studies of TCs (e.g., Möller and Montgomery 2000), we make the simplifying assumption of isothermal horizontal boundaries at $z=0$ and $\mathrm{H}$. The streamfunction and PV can then be expressed as the sum of the vertical modes. In the Boussinesq approximation in which $N^{2}$ and $\rho_{0}$ are constant with height the modal expansion is

$$
\begin{aligned}
& \psi(r, \lambda, z, t)=\sum_{m=0}^{\infty} \psi_{m}(r, \lambda, t) \cos \left(\frac{m \pi z}{H}\right) \\
& q(r, \lambda, z, t)=\sum_{m=0}^{\infty} q_{m}(r, \lambda, t) \cos \left(\frac{m \pi z}{H}\right),
\end{aligned}
$$

where $m$ is the vertical wavenumber. Substitution of (3) into Eqs. (1)-(2) yields a system of nonlinear equations for the time-dependent real amplitudes, $\psi_{m}$ and $q_{m}$ (referred to here as the equivalent barotropic (EQB) system). The nonlinear equations truncated at $m=1$ are given in appendix A. Because of the natural circular geometry of the problem, the equations are evaluated in cylindrical coordinates. For simplicity, the azimuthal mean vortex is assumed barotropic. The semispectral model described by ME98 and discussed in detail by Montgomery et al. (2000), modified to allow finite $L_{R}$, is used to perform the numerical computations (see ap- 
pendix A). A 2000-km outer radius domain with radial grid spacing $\Delta r=5 \mathrm{~km}$ and 8-mode azimuthal truncation was used. All nonlinear EQB simulations were run with a diffusion coefficient $\nu$ ranging from 100 to $200 \mathrm{~m}^{2} \mathrm{~s}^{-1}$ in order to keep the integrations stable at long times.

An advantage of the semispectral formulation is that it allows easy implementation of the linearized system of equations governing the evolution of perturbations to a circular vortex flow. Linearizing (1)-(2) about a barotropic circular vortex state, substituting (3), and Fourier decomposing $\psi_{m}$ and $q_{m}$ in azimuth yields the following linear equations for the Fourier coefficients:

$$
\begin{aligned}
\left(\frac{\partial}{\partial t}+i n \bar{\Omega}\right) \hat{q}_{m n}(r, t)-\frac{i n}{r} \frac{d \bar{\zeta}}{d r} \hat{\psi}_{m n}(r, t) & =0 \\
\frac{1}{r} \frac{\partial}{\partial r}\left(r \frac{\partial \hat{\psi}_{m n}}{\partial r}\right)-\left(\frac{n^{2}}{r^{2}}+\gamma_{m}^{2}\right) \hat{\psi}_{m n} & =\hat{q}_{m n}, \quad n \geq 1, \\
\frac{1}{r} \frac{d}{d r}\left(r \frac{d \bar{\psi}}{d r}\right) & =\bar{\zeta}
\end{aligned}
$$

where $n$ is the azimuthal wavenumber, $\gamma_{m}=m \pi / L_{R}$ is the inverse internal Rossby deformation radius for the perturbation, $\left({ }^{\wedge}\right)_{m n}$ are the Fourier coefficients, $\bar{\Omega}$ is the azimuthal-mean angular velocity, and $\bar{\zeta}$ is the azimuthal-mean geostrophic relative vorticity. The vortex evolution simulated by the linear EQB model will be compared to that simulated by the nonlinear models in sections 3 and 4 to gain insight into the nature of the alignment dynamics.

\section{Vertical alignment of a broadly distributed tilted vortex}

\section{a. Initial conditions}

In the experiments presented here the azimuthal-mean vortex will be assumed barotropic. The initial symmetric PV takes the form

$$
\bar{q}(r)=\bar{q}_{\max } e^{-(\sigma r)^{2}},
$$

where $\bar{q}_{\max }$ is the maximum mean PV and $\sigma$ is the inverse decay length of the PV profile. The mean vortex used by ME98 with $\bar{q}_{\max }=9.0 \times 10^{-5} \mathrm{~s}^{-1}$ and $\sigma^{-1}=$ $167 \mathrm{~km}$ is depicted in Fig. 1 (solid curve). The maximum wind speed is $5 \mathrm{~m} \mathrm{~s}^{-1}$ and the radius of maximum wind (RMW) is $200 \mathrm{~km}$. The dependence of the results on horizontal vortex scale and structure is discussed in appendix B.

The initial PV asymmetry has the vertical structure of the first internal baroclinic mode $(m=1)$, unless otherwise stated, and is consistent with the assumption of isothermal horizontal boundary conditions discussed in section 2. For simplicity, the mean vortex is perturbed with a single azimuthal Fourier mode, thus

$$
q^{\prime}(r, \lambda, z, t)=\hat{q}_{1 n}(r, t) \cos \left(\frac{\pi z}{H}\right) e^{i n \lambda}+\text { c.c. }
$$

where c.c. denotes the complex conjugate of the preceding quantity. In the general case $n$ can be any azimuthal wavenumber. Isolated anomalies composed of a superposition of azimuthal wavenumbers are considered in section 4 in our investigation of the merger and alignment of convectively generated PV within a preexisting vortex. For the tilted vortex simulations the horizontal structure of the PV perturbation is that of the azimuthal wavenumber $1(n=1)$ pseudomode (Michalke and Timme 1967; Weber and Smith 1993; Smith and Montgomery 1995; MK). In two-dimensional flows the pseudomode represents a vortex displacement and has the radial PV structure

$$
\hat{q}_{11}(r)=\tilde{\alpha} \frac{d \bar{q}}{d r},
$$

where $\tilde{\alpha}$ is a constant conversion factor. We define $\tilde{\alpha}=$ $\alpha \bar{q}_{\max } /(d \bar{q} / d r)_{\max }$, where $(d \bar{q} / d r)_{\max }$ is the maximum mean PV gradient and $\alpha$ is a nondimensional amplitude factor.

For a given mean vortex with horizontal scale $L$ (roughly the RMW) and vertical scale $H$ the only two adjustable parameters are $\alpha$ and $\gamma_{1}$. Variation of $\alpha$ changes the angle of inclination of the PV column from the vertical. Equations (7)-(9) best represent a tilted vortex for values of $\alpha$ much less than unity. For $\alpha>$ 0.3 higher azimuthal harmonics are included in expression (8) and a more accurate form for $\hat{q}_{11}$ is used.

Before investigating the full $\alpha$ and $\gamma_{1}$ parameter space, we begin by defining a benchmark case and comparing the vortex evolution simulated by the QG3D and EQB models. The benchmark simulation using $\alpha=0.3$ and $\gamma_{1}=3.14 \times 10^{-6} \mathrm{~m}^{-1}$ is shown in Fig. 2 in terms of PV. Midlatitude values of $f_{0}=10^{-4} \mathrm{~s}^{-1}, H=10$ $\mathrm{km}$, and $N=10^{-2} \mathrm{~s}^{-1}$ are used to define $\gamma_{1}$. To verify that (7)-(9) is a valid approximation to a tilted vortex, the QG3D model is initialized with a linearly tilted PV column having an angle of inclination from the vertical nearly identical to that of the EQB benchmark vortex. The vortex evolution simulated with the QG3D model (Fig. 2a) is replicated well by the nonlinear, truncated EQB model (Fig. 2b). The vortex precesses about the stationary midlevel centroid with no obvious sign of alignment over the $4 \tau_{e}$ period (where $\tau_{e}$ denotes a mean vortex circulation period at the RMW, which is approximately 2.9 days for this vortex). The precession period of the vortex is approximately 12.5 days, or just over $4 \tau_{e}$. As evident from comparison of the two simulations, the interaction between vertical modes is not crucial to understanding the vortex evolution for the small tilts considered here. Therefore, in our exploration of the alignment mechanism, the EQB model will be primarily used. All of the principle results to be shown, however, have been verified with the QG3D model. 
(a)
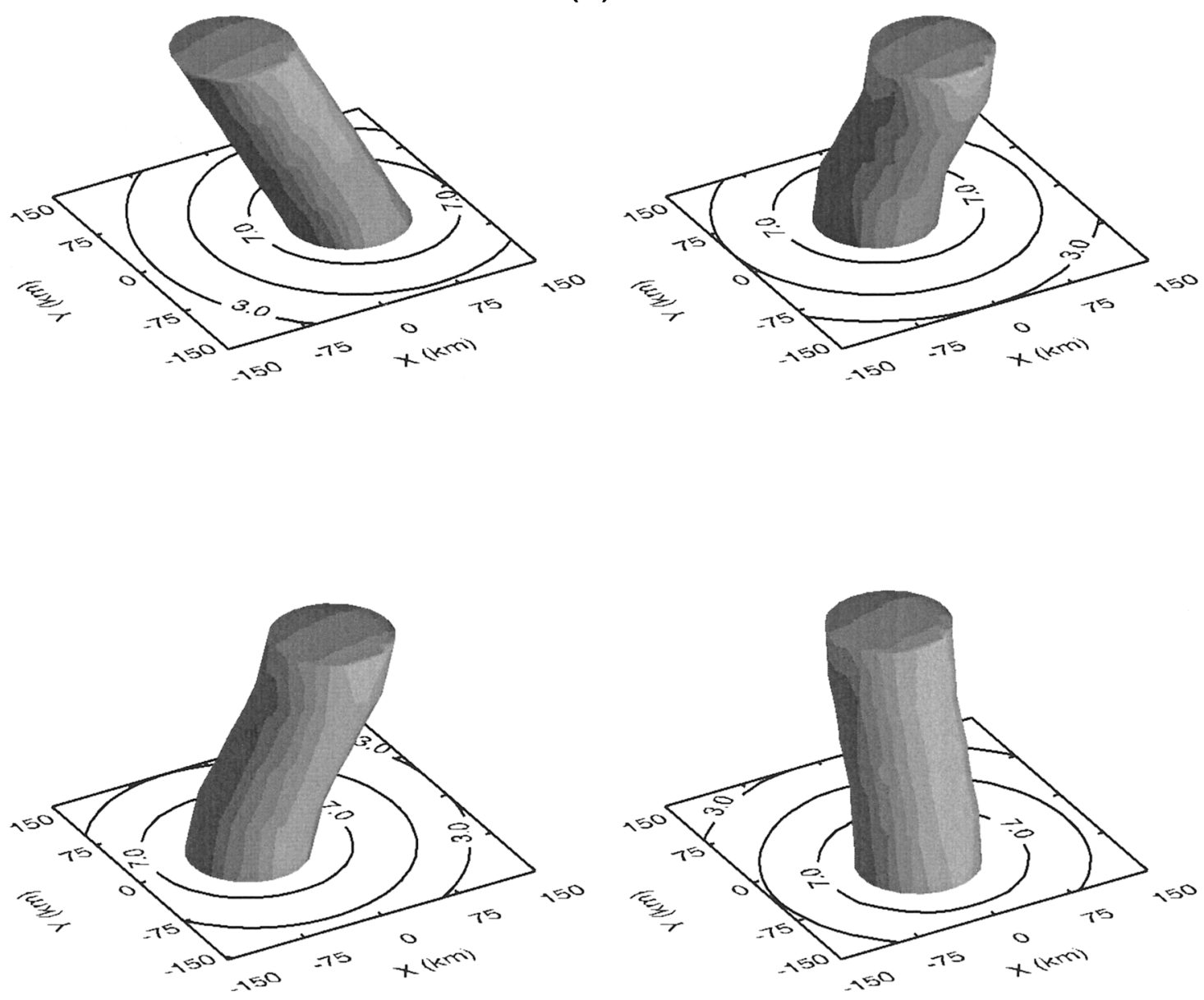

FIG. 2. Evolution of vortex PV (from left to right) at $t=0,1.5 \tau_{e}, 2.5 \tau_{e}$, and $4 \tau_{e}$ for the benchmark run with $\alpha=0.3$ and $\gamma_{1}=3.14 \times$ $10^{-6} \mathrm{~m}^{-1}$. Only the inner $300 \mathrm{~km} \times 300 \mathrm{~km}$ is shown to emphasize the vortex tilt. The vertical depth is $10 \mathrm{~km}$. Results from (a) the QG3D model with PV isosurface $8.0 \times 10^{-5} \mathrm{~s}^{-1}$ and (b) the nonlinear EQB model with PV isosurface $8.5 \times 10^{-5} \mathrm{~s}^{-1}$ are shown. The different initial conditions are described in the text. To ensure conservation of domain-integrated PV in the QG3D model the mass-weighted average of PV has been subtracted from the total field.

The dependence of the alignment process on $\alpha$ is considered below. The initial horizontal distance between upper- and lower-level PV centroids $\left(d_{i}\right)$ defined by

$$
d_{i}=2\left[\left(\frac{\int_{A} x q d A}{\int_{A} q d A}\right)^{2}+\left(\frac{\int_{A} y q d A}{\int_{A} q d A}\right)^{2}\right]^{1 / 2}
$$

(where the integral over the domain area A can be evaluated at either the upper or lower level due to the mirror symmetry about the middle level for perturbations representing a tilted vortex) is listed in Table 1 for each of the primary simulations. The midlatitude value of $\gamma_{1}=$ $3.14 \times 10^{-6} \mathrm{~m}^{-1}$ is used in all simulations, except in section $3 \mathrm{c}$ where the $\gamma_{1}$-dependence of vortex alignment is explored.

\section{b. Linear vortex Rossby wave dynamics}

A useful diagnostic in the study of vortex merger and vortex axisymmetrization on a vortex with monotonically decreasing basic-state vorticity is the vortex beta Rossby number, $R_{\beta}$ (Möller and Montgomery 2000; Enagonio and Montgomery 2001). Mathematically it is defined as the ratio of the nonlinear terms in the PV equation to the effective "beta" term involving the mean PV gradient of the basic-state vortex. Whereas the vortex itself is the perturbation in the problem of vortex motion on a $\beta$ plane (McWilliams and Flierl 1979; Montgomery et al. 1999), here the perturbation is the departure from vertical alignment. In studies of vortex motion the beta Rossby number is large compared to unity (a large "perturbation"). The tilted vortices considered here are characterized by beta Rossby numbers less than unity (a small "perturbation"). In the nondivergent limit the vortex beta Rossby number scales as 
15 August 2001

REASOR AND MONTGOMERY

2311

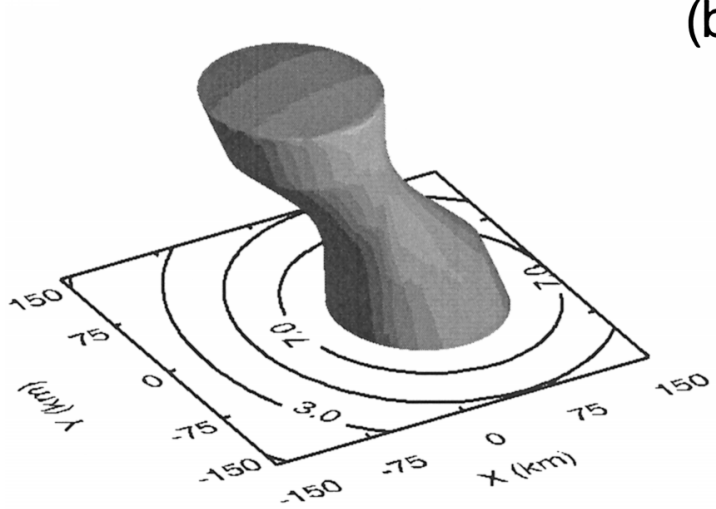

(b)
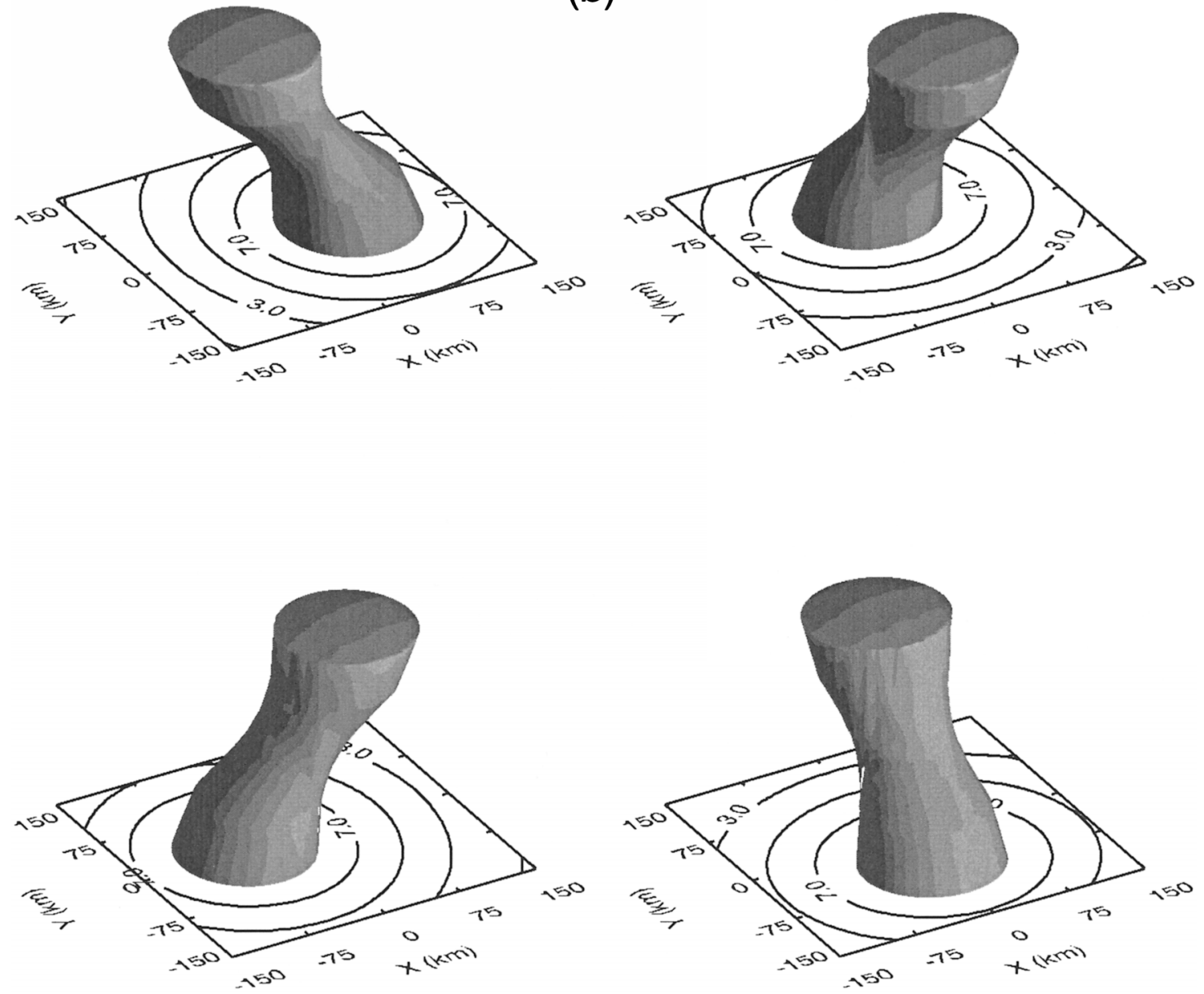

Fig. 2. (Continued)

$$
R_{\beta} \sim \frac{V^{\prime}}{L^{\prime 2} \frac{d \bar{\zeta}}{d r}},
$$

where $V^{\prime}$ is the perturbation velocity amplitude, $L^{\prime}$ the perturbation horizontal scale, and $\bar{d} \bar{\zeta} / d r$ the radial varticity gradient of the basic-state vortex. $R_{\beta}$ provides a measure of how important nonlinear advection is compared to the vortex Rossby wave restoring mechanism. For $R_{\beta}$ much less than unity perturbations on an everywhere monotonic mean vortex are expected to disperse as vortex Rossby waves. The wave-mean interpretation of MK usefully and accurately characterizes the dynamiss in this parameter regime.

In previous work unforced vortex alignment has been described in much the same way as vortex merger in two dimensions: The flow induced by the upper-level PV anomaly adverts the lower-level anomaly, and vice versa. Attempts to predict the evolution of tilted vortices given the initial vertical tilt and deformation radius have focused on finding solutions to the nonlinear equations of motion. We agree that this approach is the correct one for upper- and lower-level anomalies initially seporated by large distances. But is this way of viewing the problem the most insightful one for upper and lowerlevel anomalies separated by small distances? We propose that the asymptotic dynamics (i.e., when the vartices are close enough together) of vortex merger and alignment in three dimensions is more usefully viewed as linear. This is not to say that nonlinear advection is identically zero, but rather that its role in the evolution of vertices with small but finite amplitude tilts is secondary to linear advection. The approach taken here is to begin with an aligned vortex and systematically increase the vertical tilt. With each progressive increase in the separation between upper- and lower-level nomalien we ask the question: To what extent is the subsequent vortex evolution described by linear dynamics?

A schematic illustration of what is meant by linear dynamics is shown in Fig. 3. The tilted PV column is decomposed into an azimuthal mean, $\bar{q}$, which for simplicity is assumed barotropic, and a departure from that mean, $q^{\prime}$. In the linear approximation, $\bar{q}$ at upper (lower) levels is radially advected by the perturbation wind field 

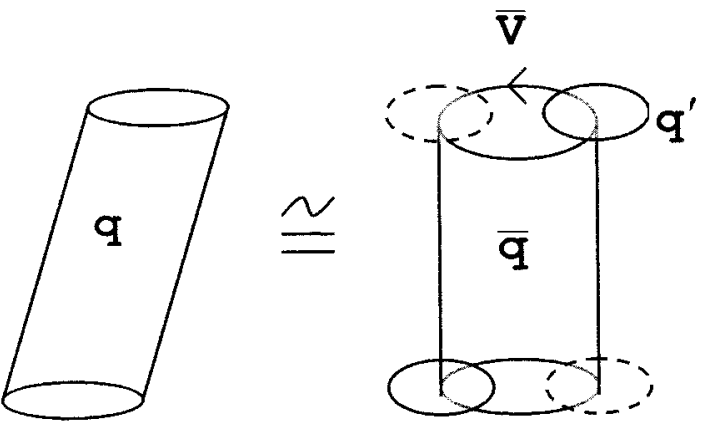

FIG. 3. Schematic illustration of the linear vortex alignment mechanism. A PV column bounded by rigid lids at $z=0$ and $\mathrm{H}$ is tilted from west to east with height. For small tilts this configuration is decomposed into an azimuthal-mean barotropic vortex (with tangential wind and $\mathrm{PV}, \bar{v}$ and $\bar{q}$, respectively) and an azimuthal wavenumber 1 asymmetry with vertical structure of the first internal baroclinic mode. The tilted vortex evolution is then governed by the interaction of the perturbation with the mean flow.

associated with the upper- (lower) level PV anomaly and, depending on the magnitude of the vertical penetration depth of the vortex flow, $f_{0} L / N$, the lower- (upper) level PV anomaly. It is this radial advection in conjunction with the azimuthal advection of $q^{\prime}$ by the mean tangential wind, $\bar{v}$, that governs the evolution of the tilted vortex in the linear approximation. Of course in the limit of large vertical tilt (and presumably large $R_{\beta}$ ), $q^{\prime}$ approaches the magnitude of $\bar{q}$, and linear theory will no longer be valid. Our intent is to provide physical insight into the vortex alignment process at small vertical tilts and to then illustrate the range of applicability of the linear ideas.

For the benchmark run with $\alpha=0.3, R_{\beta}$, computed using the strict mathematical definition stated above, is found to be approximately 0.25 in the vicinity of the PV perturbation maximum. Figure 4 shows the total and wavenumber one PV at the surface $(z=0)$ from the linear and nonlinear EQB models. Although $R_{\beta}$ is not infinitesimally small in this case, good agreement between the linear and nonlinear simulations is nevertheless observed. Both vortex simulations show radially propagating, sheared vortex Rossby waves superposed on a quasi-discrete wavenumber $1 \mathrm{PV}$ feature. The radial vortex Rossby wave propagation is illustrated in Fig. 5 for the linear simulation. As predicted by MK, the radial propagation speed of the wave packets decreases in time as the waves are sheared to finer and finer radial scales. The quasi-discrete wavenumber one PV asymmetry near $r=125 \mathrm{~km}$ persists over the $5 \tau_{e}$ period with only a $10 \%-20 \%$ decrease in amplitude. Consistent with a quasi-discrete vortex Rossby wave it propagates cyclonically around the vortex at a speed less than the local mean tangential wind. Figure 4 also shows the PV evolution for the same initial vortex using the QG3D model. The azimuthal propagation and structure of the wavenumber $1 \mathrm{PV}$ asymmetry are virtually identical in the two nonlinear simulations. Both nonlinear simulations also show less sheared vortex Rossby wave propagation than the linear simulation.

Figure 6 shows the domain-integrated perturbation energy (kinetic and available potential) contained in wavenumber 1 . The linear solution indicates only a $30 \%$ decrease in energy over the $5 \tau_{e}$ period. Most of the initial asymmetry energy is trapped in the quasi-discrete vortex Rossby wave, which slowly loses its energy through the radial propagation of sheared vortex Rossby waves. Due to wave-wave interactions the nonlinear solution shows a more modest decrease in wavenumber 1 energy of $5 \%-10 \%$. The use of the pseudomode to represent the initial horizontal structure of wavenumber $1 \mathrm{PV}$ is fortuitous since it projects strongly onto the quasi-discrete vortex Rossby wave. A general initial condition will tend to project more onto the sheared vortex Rossby waves, obscuring the quasi-discrete structure at early times. As demonstrated by Smith and Montgomery (1995, and references therein) and later extended by MK to account for the Rossby wave effects, the energy contained in the sheared Rossby waves will ultimately diminish with time. Thus, the quasi-discrete wave structure will eventually emerge. It is interesting to note that higher wavenumbers show a markedly different behavior than wavenumber 1 with the energy falling to near zero after only a $1-2 \tau_{e}$ period. We conclude that wavenumber 1 is unique within the parameter regime under consideration. The corotation resulting from the long-lived propagation of the wavenumber 1 asymmetry observed in the nonlinear simulations is reproducible using linear dynamics alone, validating the conceptual model illustrated in Fig. 3 for finite-amplitude vortex tilts.

To determine the range of $\alpha$ for which linear vortex Rossby wave theory captures the essence of the alignment process we compare the intercentroid separation distance between upper- and lower-level PV anomalies, $d_{i}$, predicted by the linear and nonlinear EQB models. Figure 7a shows the trajectories of the upper- and lowerlevel centroids for the benchmark case. In both simulations the centroid makes slightly more than one orbit at a radius of approximately $60 \mathrm{~km}$ during the $5 \tau_{e}$ period. The discrepancy between the linear and nonlinear predictions of $d_{i}$ increases to about $5 \mathrm{~km}$ during the period, as shown in Fig. 7b. The nonlinear prediction shows a reduced rate of alignment, although we would argue that the linear dynamics is still capturing the essence of the corotation and slow alignment of upper- and lower-level PV anomalies.

For an even smaller tilt amplitude of $\alpha=0.1$, little discrepancy between linear and nonlinear simulations is found. In this case $R_{\beta} \approx 0.08$, which confirms the negligible role played by nonlinear advection. The quasidiscrete vortex Rossby wave has essentially the same structure and propagation speed in both simulations (not shown).

To simulate a vortex with more exaggerated tilt the EQB model is initialized with the mean vortex given 

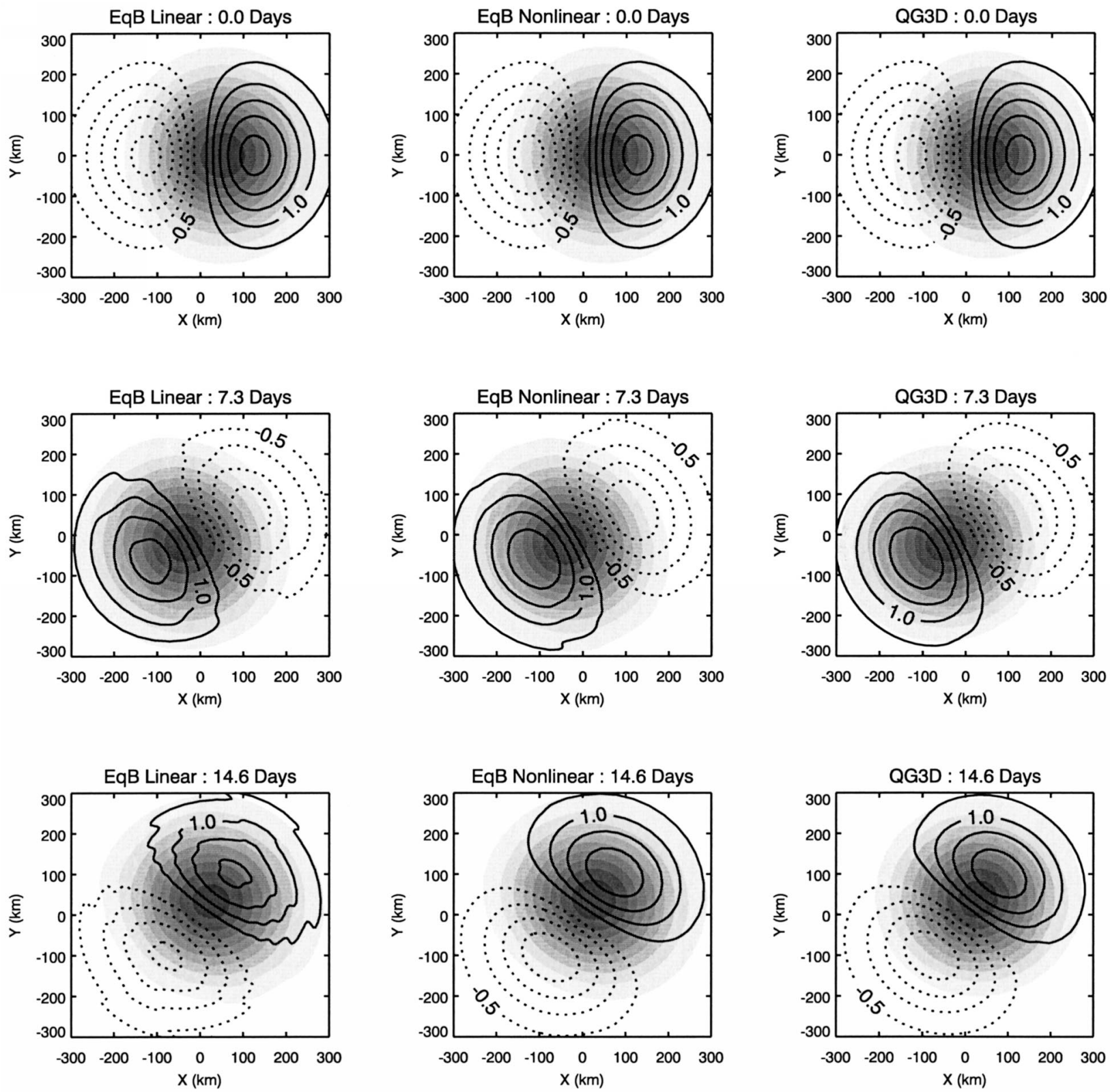

FIG. 4. Evolution of total vortex PV (shaded) and wavenumber 1 component of PV (contour interval $0.5 \times 10^{-5} \mathrm{~s}^{-1}$ with negative values dashed) at $z=0$ over a $5 \tau_{e}$ period for the benchmark run (see Fig. 2). From left to right are shown the results from the linear EQB, nonlinear EQB, and QG3D models, respectively. Aside from the PV correction in the QG3D model, the initial conditions are identical.

by (7), but displaced $100 \mathrm{~km}$ to the east of the polar coordinate system origin at $z=0$, and tilted linearly with height to the west. An azimuthal and vertical wavenumber decomposition of this initial condition shows that only four modes need to be included in the EQB initialization: $(m, n)=(0,0),(1,1),(2,0)$, and $(0,2)$. In keeping with the assumption of a barotropic mean vortex, the $n=0$ component of the vortex at $z=0$ is used at all levels. For this case the initial upper- and lower-level PV centroids are separated by $175 \mathrm{~km}, \alpha \approx$ 0.5 and $R_{\beta} \approx 0.5$.
Figure 8 shows the evolution of total and wavenumber $1 \mathrm{PV}$ from the linear and nonlinear EQB models. Also shown is the PV at the lowest level of the QG3D model for a vortex tilted linearly with height (without the above approximations). The two nonlinear simulations basically agree, confirming the utility of the truncated equivalent barotropic approach even for relatively large vortex tilts. More important is the similarity between the linear and nonlinear simulations. Although there is considerably more radial vortex Rossby wave dispersion in the linear simulation, the rotation frequencies of the low- 


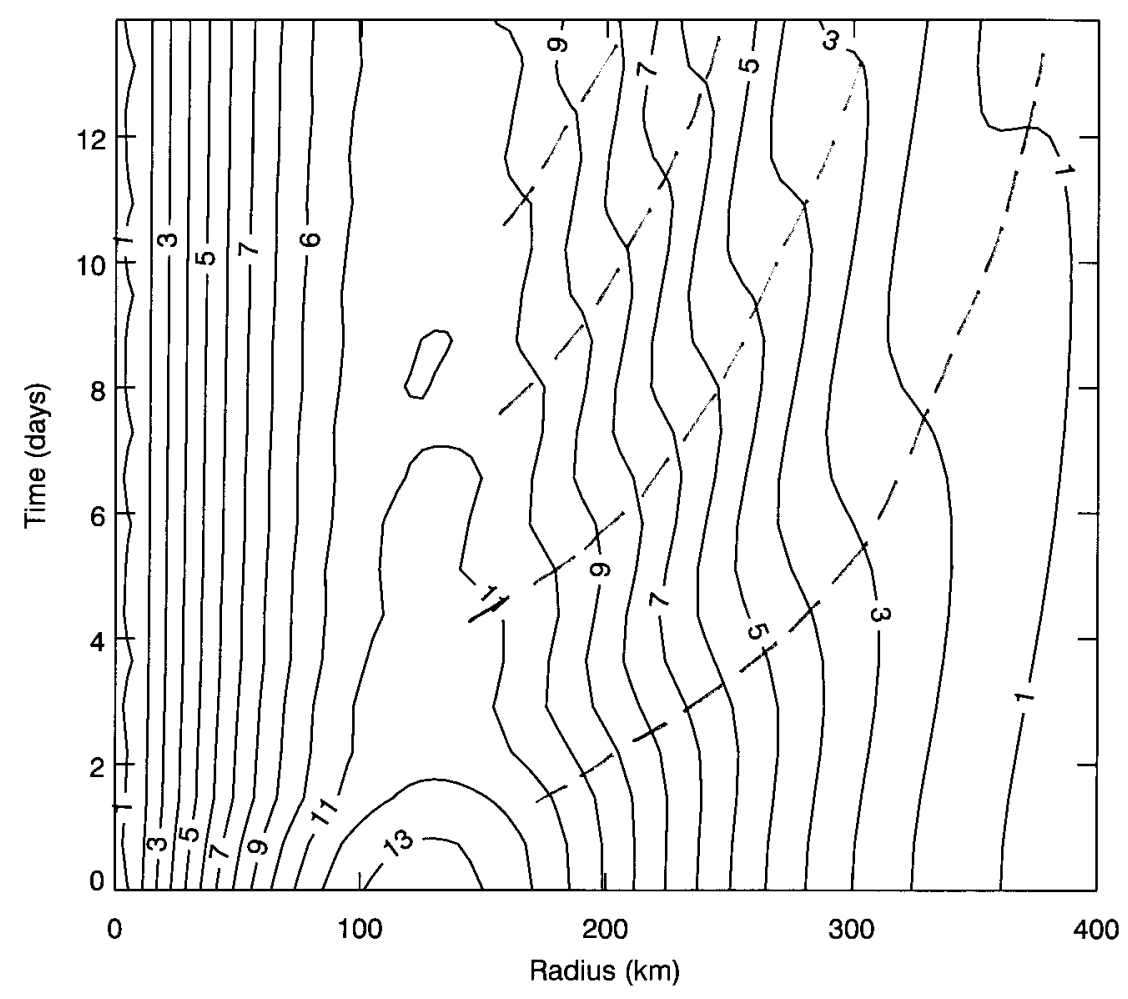

FIG. 5. Radius-time plot of the wavenumber $1 \mathrm{PV}$ amplitude at $z=0$ over a $5 \tau$ period for the linear benchmark run. The dashed lines are phase lines highlighting the outward propagation of vortex Rossby wave packets in time. The quasi-discrete vortex Rossby wave persists near $125-\mathrm{km}$ radius. Contour interval is $1.0 \times 10^{-6} \mathrm{~s}^{-1}$.

level vortex about the midlevel centroid are virtually the same, as shown in Fig. 9. The details of the $d_{i}$ evolution over $5 \tau_{e}$ and departure from that shown in Fig. 7 can be attributed to the greater projection of the initial PV asymmetry onto sheared vortex Rossby waves. Overall the linear dynamics still captures the essence of the tilted vortex evolution. Thus, while nonlinear effects increase in importance with increasing $R_{\beta}$ and modify the vortex structure (i.e., counteract the sheared vortex Rossby wave dispersion), the underlying quasi-discrete vortex Rossby wave persists in dominating the solution.

These results suggest a new interpretation for the corotation of vertically separated, overlapping vortices for internal Rossby deformation radii greater than the horizontal scale of the vortex. Polvani (1991) explained the presence of corotation geometrically as a consequence of the initial vortex configuration being near a geostrophic PV equilibrium (i.e., a stable V state). Here the corotation of continuously distributed vortices is attributed to the cyclonic propagation of a quasi-discrete wavenumber 1 vortex Rossby wave. Analytical solutions to the linear QG equivalent barotropic problem are currently unavailable for continuous PV distributions, but may present themselves for special cases. Such a solution would allow one, for example, to predict the linear corotation frequency. For now we will continue to explore this linear interpretation of the alignment process, considering the effect of varying $\gamma_{1}$.

\section{c. Dependence on internal deformation radius}

Varying $\gamma_{1}$ can be viewed in terms of changing the depth of the vortex, the static stability, or the planetary vorticity. The dependence of vortex alignment on vortex depth has been explored in recent studies motivated by observations from QG free-decay turbulence simulations (McWilliams 1989; Viera 1995; Dritschel and de la Torre Juárez 1996). The tilted vorticity configurations in these studies attain equilibrium at certain vertical scales. Moist convection will increase $\gamma_{1}$ by reducing the static stability (Emanuel et al. 1987; Montgomery and Farrell 1992). As further discussed in section 4, for more rapidly swirling flows, $f_{0}$ in the expression for $\gamma_{1}$ is replaced by the geometrical mean of the modified Coriolis parameter and absolute vorticity associated with the basic-state circular vortex (Shapiro and Montgomery 1993). We may then anticipate the vortex evolution for more rapidly swirling flows by increasing $f_{0}$ in the QG formulation.

The dependence of vertical alignment on internal deformation radius and horizontal vortex scale is known from QG contour dynamics (CD) model simulations (Polvani 1991). For horizontal vortex scales greater than 


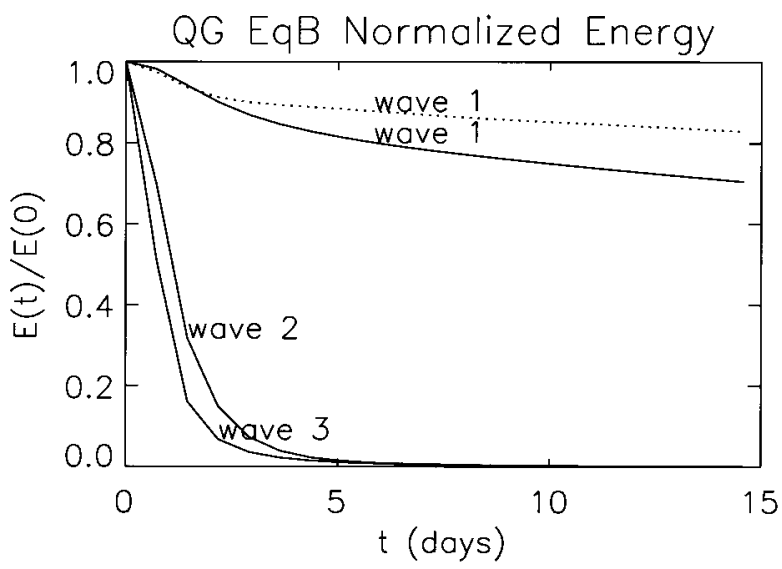

FIG. 6. The QG equivalent barotropic volume-integrated energy contained in wavenumber 1 from the nonlinear (dashed) and linear (solid) EQB benchmark runs (see Fig. 2) as a function of time. Also shown is the linear energy evolution for initial conditions with the pseudomode radial structure, but azimuthal structure of wavenumbers 2 and 3. The energy is normalized by its initial value in all cases.

the internal deformation radius the vertically tilted vortex tends toward alignment, while for smaller vortex scales alignment is inhibited and corotation is observed. The linear interpretation of the alignment mechanism can help explain why this is so.

In the benchmark simulation (see Fig. 2) we found that alignment was inhibited and the vortex precessed about its midlevel centroid with a small increase in the discrepancy between linear and nonlinear simulations with time out to several $\tau_{e}$. Figure 10 shows the evolution of the same tilted vortex, but for larger $\gamma_{1}=20.0$ $\times 10^{-6} \mathrm{~m}^{-1}$. The QG3D (not shown) and nonlinear EQB models show nearly identical results, so we focus on the vortex evolutions simulated by the EQB linear and nonlinear models. In both simulations alignment of the tilted vortex and attendant filamentation of PV are observed, as found in previous studies using vortex patches (Polvani 1991; Viera 1995; Dritschel and de la Torre Juárez 1996). The linear results confirm that the filamentation observed in the nonlinear simulation near the core of the stable vortex monopole is primarily a consequence of linear vortex Rossby wave dynamics and not nonlinear mixing or "wave breaking." Figure 11 shows that in both simulations the vortex undergoes complete alignment within $5 \tau_{e}$ at virtually identical rates. In studies using a single vortex patch the filamentation is a nonlinear process. Therefore, there is a fundamental difference between the physical mechanisms responsible for axisymmetrization (or alignment in our case) for smooth and piecewise-continuous vortices (Smith and Montgomery 1995; Bassom and Gilbert 1998). We believe the linear mechanism is more relevant to TC-like vortices in the limit of small vertical tilts.

The increased agreement between linear and nonlinear simulations for this larger value of $\gamma_{1}$ can be understood by examining the $\gamma_{1}$-dependence of $R_{\beta}$. Figure
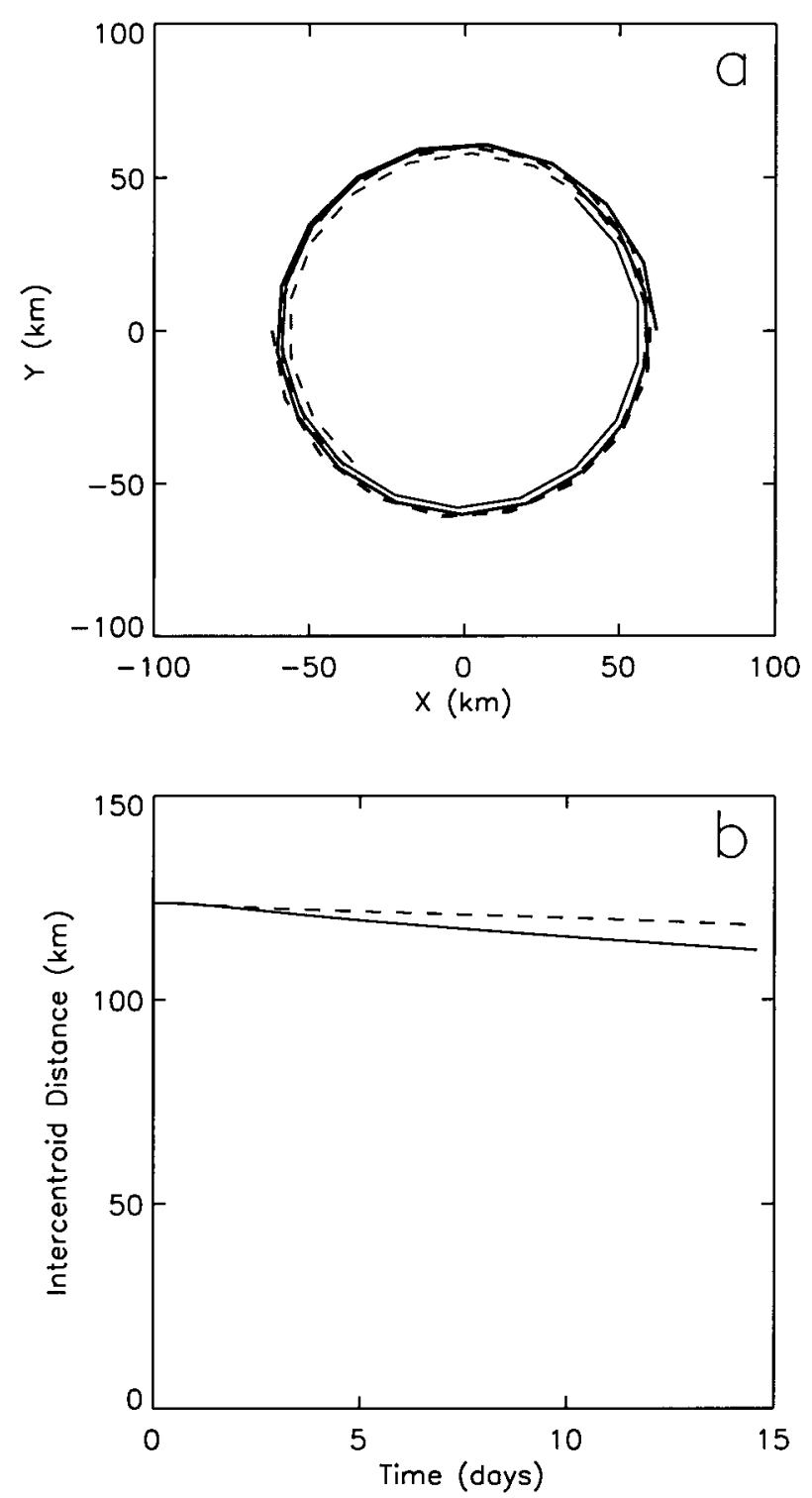

FIG. 7. Evolution of the EQB PV intercentroid separation between $z=0$ and $\mathrm{H}$ over $5 \tau_{e}$ for the benchmark run (see Fig. 2). (a) Trajectory of the PV centroid at upper (solid) and lower (dashed) levels. The heavy lines show the nonlinear evolution, while the fine lines show the linear evolution. (b) Time series of intercentroid separation distance from the linear (solid) and nonlinear (dashed) models.

12 shows the initial $R_{\beta}$ in the vicinity of the maximum perturbation PV for various $\alpha$ as a function of $\gamma_{1}$. In section $3 \mathrm{~b}$ it was shown that for a given value of the internal deformation radius, $R_{\beta}$ decreases with decreased perturbation amplitude (recall $R_{\beta} \sim V^{\prime}$ ) leading to increased agreement between linear and nonlinear simulations. The naive scaling (11) would not predict a dependence on $\gamma_{1}$, contrary to Fig. 12, which clearly shows $R_{\beta}$ decreasing with increasing values. Farge and Sadourny (1989) explained the dependence of their QG shallow water turbulence simulations on deformation radius from the perspective of energy and enstrophy 

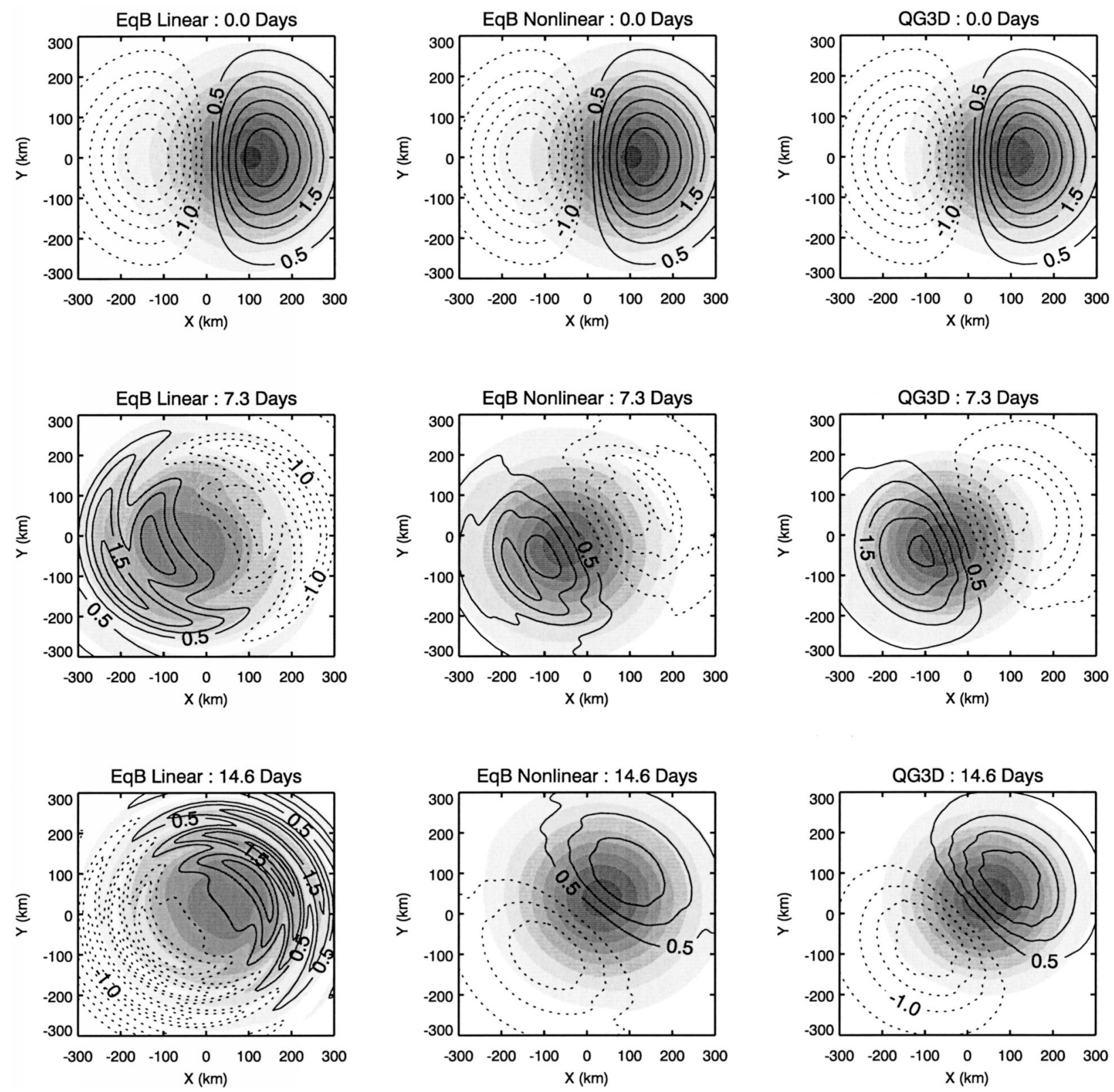

FIG. 8. As in Fig. 4 but for $\alpha \approx 0.5$. See text for details on the initial conditions.

spectra. For large rotation rates (i.e., large $\gamma_{1}$ ) they demonstrated that the cascade of potential enstrophy to smaller scales is inhibited. This behavior can also be explained geometrically by examining the dependence of the QG (baroclinic) perturbation PV inversion on $\gamma_{1}$. For small $\gamma_{1}$, the Green function decays slowly with radius and PV over a broad area is incorporated into the inversion for streamfunction at a point. In this case the streamfunction field is unable to replicate strong curvature of the PV field. For much larger $\gamma_{1}$, the Green function decays rapidly with radius and the streamfunction field is better able to reproduce the curvature of the PV field over the entire domain, leading to near- parallel PV and streamline contours as illustrated in Fig. 13 , that is,

$$
J\left(\psi^{\prime}, q^{\prime}\right) \rightarrow 0 \text { as } \gamma_{1} \rightarrow \infty .
$$

Thus, for $\gamma_{1} \rightarrow \infty, R_{\beta}$ tends to zero and the linear and nonlinear simulations converge. An additional consequence of this dependence of $R_{\beta}$ on $\gamma_{1}$ is that the linear vortex Rossby wave dynamics will accurately capture the alignment process for a wider range of amplitudes the larger $\gamma_{1}$ is. Although our focus here is on small to moderate tilts from the vertical, even vortices for which $d_{i} / L \sim 2$ (i.e., the RMWs of the upper and lower vortices 

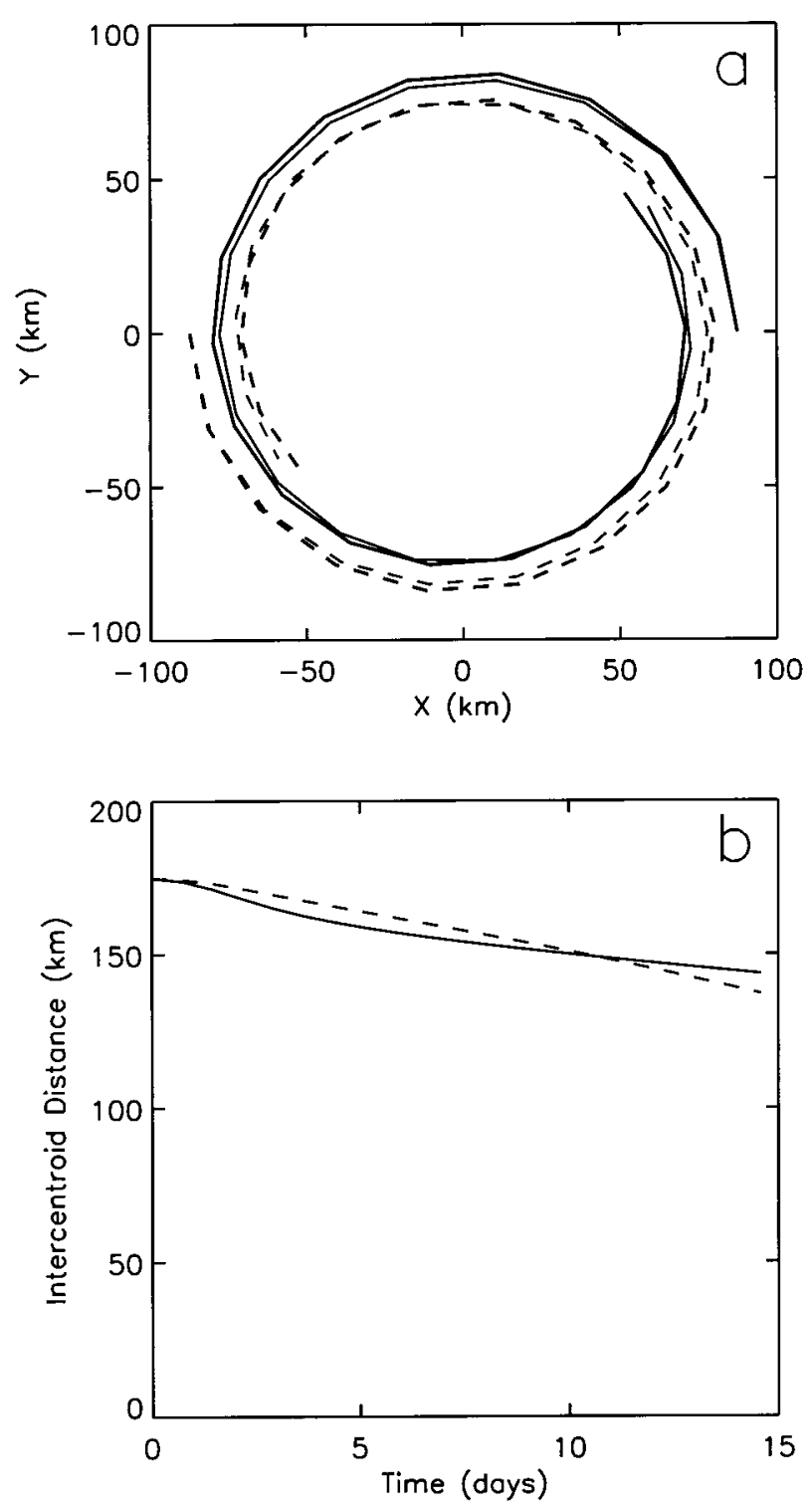

FIG. 9. As in Fig. 7 but for $\alpha \approx 0.5$.

just overlap) the alignment process could still fall within the linear regime as long as $\gamma_{1}$ is large enough.

The complete picture of the dependence of vortex alignment on $\gamma_{1}$ for small initial tilts $(\alpha=0.1)$ is shown in Fig. 14. The intercentroid distance after $5 \tau_{e}$ for both linear and nonlinear simulations is plotted as a function of $\gamma_{1}$. As expected, no discernible difference between the simulations is observed. After $10 \tau_{e}$ the curve is essentially the same, except the transition region between small and large $\gamma_{1}$ is steeper (not shown). Three regions are clearly identifiable in the $\gamma_{1}$ phase space.

For $\gamma_{1} \geq 10.0 \times 10^{-6} \mathrm{~m}^{-1}$ complete vertical alignment takes place. The corresponding threshold internal deformation radius is $100 \mathrm{~km}$ which is smaller than the scale of the current vortex. The presence of vertical alignment at small $\alpha$ (or equivalently small initial $d_{i}$ ) should be contrasted with the two-layer CD results of Polvani (1991). There, corotation was observed for all $\gamma_{1}$ in this range. As discussed in appendix $\mathrm{C}$, this is a consequence of using a single PV interface in the CD model. Dritschel (1998) found that sufficiently steepedged vortices in two-dimensional flows can also resist axisymmetrization, but such vortices are not typically observed in the weak TC context. Utilizing the fact that the dynamics is fundamentally linear in this regime we can demonstrate why continuously distributed vortex alignment must occur for all small initial $d_{i}$. The argument is essentially that when $\gamma_{1}$ is large, the effective "beta" term in the linearized PV equation can be neglected. The perturbation PV to leading order is then materially conserved following the local mean tangential winds, leading to simple spirals around the vortex and algebraic decay of the perturbation streamfunction in the limit of long times (see appendix $\mathrm{C}$ for details). It is the sheared vortex Rossby waves which provide the irreversible deformation of the PV field needed for alignment.

The small-amplitude version of the benchmark run $\left(\gamma_{1}=3.14 \times 10^{-6} \mathrm{~m}^{-1} ; \alpha=0.1\right)$ falls within the transition zone in what we call the slowly aligning regime. The horizontal vortex scale lies close to the midpoint of this regime. It should be noted that the vortex does not actually align here according to our definition which requires $d_{i}=0$. At longer times this transition region becomes narrower. Vortices at the large $\gamma_{1}$ end of the transition region enter into the alignment regime after a sufficiently long period of time, while little change in the structure of the curve at small $\gamma_{1}$ is ever observed during longtime integrations of the model.

The behavior of a tilted vortex for which $\gamma_{1} \leq 2.5$ $\times 10^{-6} \mathrm{~m}^{-1}$ is unlike that found in the rest of the $\gamma_{1}$ phase space. Recall that the initial value of $d_{i}$ is approximately $41 \mathrm{~km}$ (see Table 1 ). Thus, there is virtually no tendency for alignment (even after $10 \tau_{e}$ ) in this regime. The vortex corotation is accounted for by the azimuthal propagation of the quasi-discrete vortex Rossby wave first noted in the benchmark simulation. In the limit of vanishing $\gamma_{1}$ (the nondivergent limit) the stationary pseudomode discussed by MK is recovered. As $\gamma_{1}$ is increased from the nondivergent limit, the longtime radial structure of azimuthal wavenumber 1 PV still closely resembles the pseudomode, but rotates at a nonzero frequency. If $\gamma_{1}$ becomes too large, the quasi-discrete feature ceases to exist, as discussed above. The origin and characteristics of this quasi-discrete vortex Rossby wave are discussed below.

Figure 14 also shows the dependence of $d_{i}$ on $\gamma_{1}$ for $\alpha=0.3$. There is a more noticeable discrepancy between linear and nonlinear simulations for this larger value of tilt, although the difference is small. The tilted vortex evolution is still captured by linear dynamics for all values of $\gamma_{1}$. While a decrease in discrepancy with increasing $\gamma_{1}$ in the alignment regime is expected based on vanishing $R_{\beta}$, the decrease in discrepancy with de- 


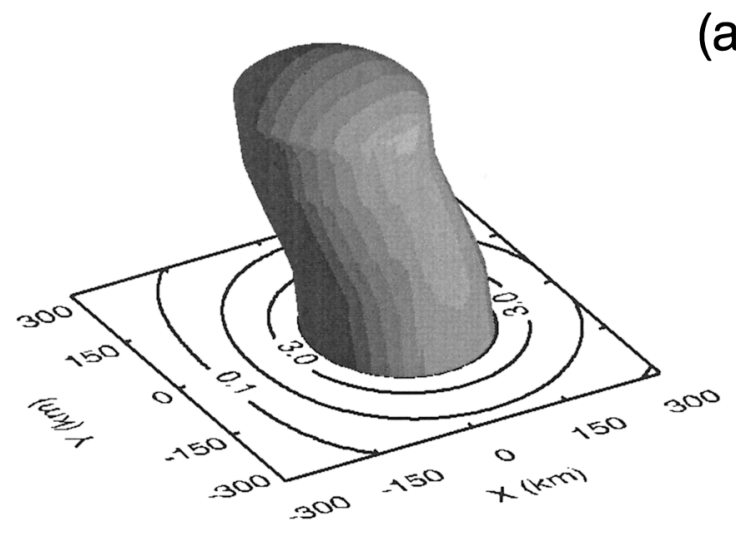

(a)
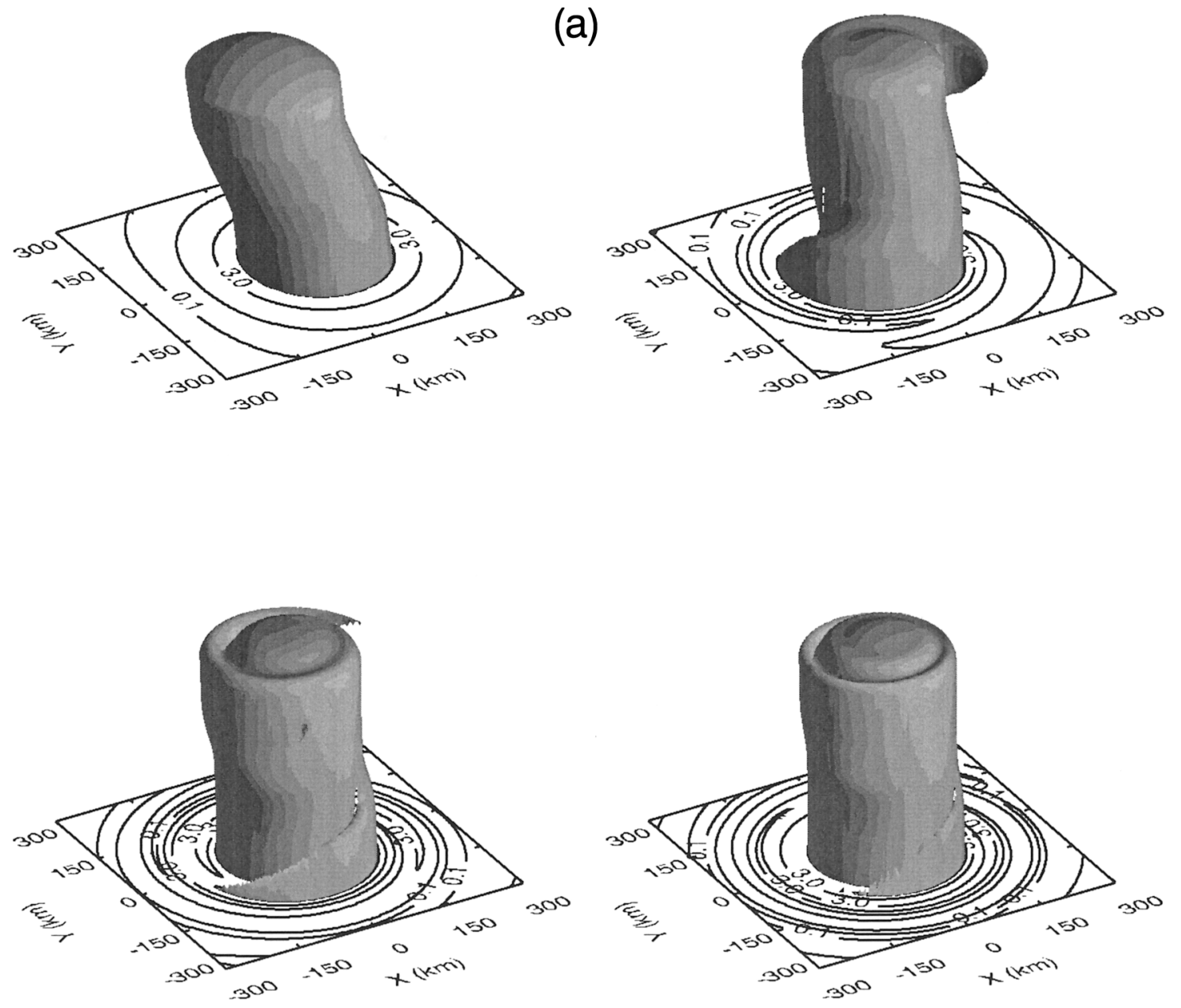

FIG. 10. Evolution of vortex PV (from left to right) at $t=0,1.5 \tau_{e}, 2.5 \tau_{e}$, and $4 \tau_{e}$ for $\alpha=0.3$ and $\gamma_{1}=20.0 \times 10^{-6} \mathrm{~m}^{-1}$. Only the inner $600 \mathrm{~km} \times 600 \mathrm{~km}$ is shown to emphasize the vortex tilt. Results from (a) the nonlinear EQB model with PV isosurface $5.0 \times 10^{-5} \mathrm{~s}^{-1}$ and (b) the linear EQB model with PV isosurface $5.0 \times 10^{-5} \mathrm{~s}^{-1}$ are shown. The contours shown are $(0.1,1,3,5) \times 10^{-5} \mathrm{~s}^{-1}$.

creasing $\gamma_{1}$ in the strict nonalignment regime was not entirely anticipated. We believe that the continued good agreement between linear and nonlinear simulations in the latter regime is a consequence of the robustness of the quasi-discrete vortex Rossby wave.

\section{d. Quasi-mode interpretation of three-dimensional vortex corotation}

For an initially tilted vortex satisfying $R_{\beta}<1$ and small $\gamma_{1}$, the longtime solution is an azimuthal wavenumber 1 asymmetry with vertical structure of the first internal baroclinic mode propagating on the mean vortex. Although the rotation rate of the asymmetry is approximately constant, and the radial structure appears largely unaffected by differential rotation, the slow, almost imperceptible decay of the asymmetry for $\gamma_{1} \leq$ $2.5 \times 10^{-6} \mathrm{~m}^{-1}$ suggests that it is not a discrete or neutral mode. In the nondivergent limit wavenumber 1 does in fact become a smooth stationary mode, but it loses this modal characteristic once the Rossby deformation radius becomes finite. To understand this behavior of wavenumber 1 , consider stable solutions to Eq. (4) of the form

$$
\hat{\psi}_{m n}(r, t)=\hat{\phi}_{m n}(r) e^{-i \omega_{m n} t}
$$

where $\omega_{m n}$ is a constant rotation frequency. Substitution into Eq. (4) yields

$$
\begin{aligned}
& \frac{d^{2} \hat{\phi}_{m n}}{d r^{2}}+\frac{1}{r} \frac{d \hat{\phi}_{m n}}{d r}-\left(\frac{n^{2}}{r^{2}}+\gamma_{m}^{2}\right) \hat{\phi}_{m n}-\frac{n d \bar{\zeta} / d r}{r\left(n \bar{\Omega}-\omega_{m n}\right)} \hat{\phi}_{m n} \\
& \quad=0 .
\end{aligned}
$$

This is an eigenvalue problem with eigen-streamfunction amplitudes, $\hat{\phi}_{m n}$, and corresponding eigenfrequencies, $\omega_{m n}$. In the nondivergent case an exact solution to (14) for $n=1$ is $\hat{\phi}=c \bar{v}$ with $\omega=0$, where $c$ is a constant (Michalke and Timme 1967). This is the aforementioned stationary pseudomode solution. For higher 

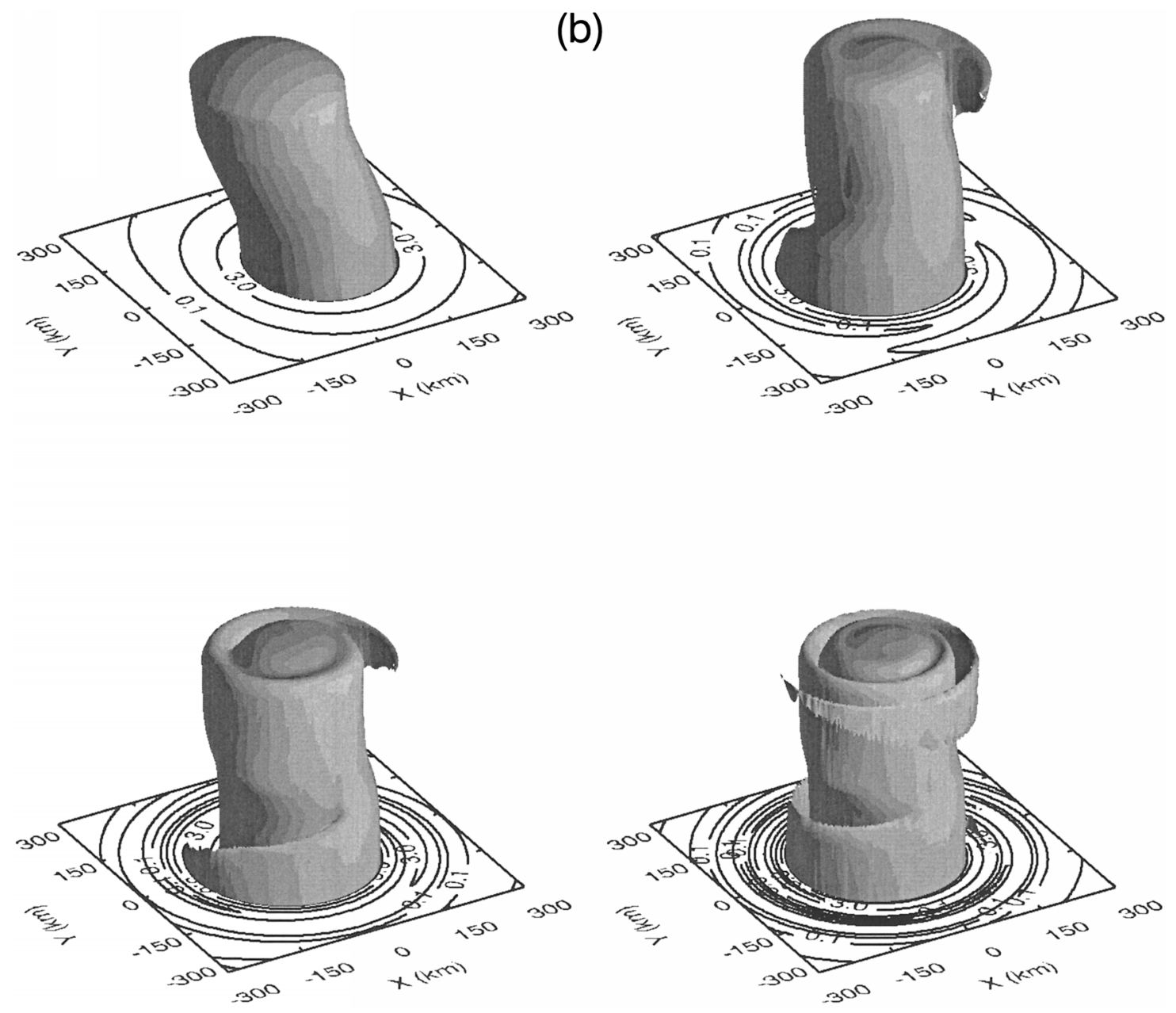

FIG. 10. (Continued)

azimuthal wavenumber perturbations to a two-dimensional vortex with monotonically decreasing mean vorticity, there are no smooth eigenfunction solutions. Only singular eigenfunction (continuous spectrum) solutions exist, that decay to zero algebraically in the limit of long times (Sutyrin 1989; Carr and Williams 1989; Smith and Montgomery 1995; MK; Bassom and Gilbert 1998). Thus, based on two-dimensional axisymmetrization experiments involving continuously distributed mean vortices like the Gaussian, one might not expect a long-lived asymmetry to be supported in three dimensions.

According to the three-dimensional QG numerical simulations at small $\gamma_{1}$, the rotation frequency of wavenumber 1 is small, but nonzero. The intrinsic frequency, $\bar{\Omega}-\omega$, will therefore pass through zero at some large but finite value of $r$. Since the simulated wavenumber 1 streamfunction is not observed to pass through zero and $d \bar{\zeta} / d r$ is nonzero for all $r$ away from the origin and $r=\infty$, solutions to (14) must reside in the continuous spectrum of discrete singular eigenfunctions. Why, then, do we observe a smooth, long-lasting wavenumber 1 asymmetry in the numerical simulations?

An answer is found through close examination of the $(m, n)=(1,1)$ eigenfunctions of $(14)$ for the benchmark mean vortex (7) and $\gamma_{1}=1.25 \times 10^{-6} \mathrm{~m}^{-1}$. The eigensolver following Gent and McWilliams (1986) and discussed by Reasor et al. (2000) is used, modified so as to permit finite deformation radius. Figure 15 shows the PV eigenfunctions surrounding the eigenfunction whose eigenfrequency is closest to the numerically simulated wavenumber 1 rotation frequency. A grouping of eigenfunctions with slightly different eigenfrequencies is found centered on the numerically observed rotation frequency with both a smooth structure and small-amplitude singular spike. The smooth structure is identical to that found in the numerical simulation at long times. The spikes occur at critical radii where the intrinsic frequency associated with each eigenfunction equals zero and (14) becomes singular. Although there is a continuum of these singular eigenfunctions for frequencies between $\bar{\Omega}_{\text {min }}$ and $\bar{\Omega}_{\text {max }}$ (Case 1960), only the 

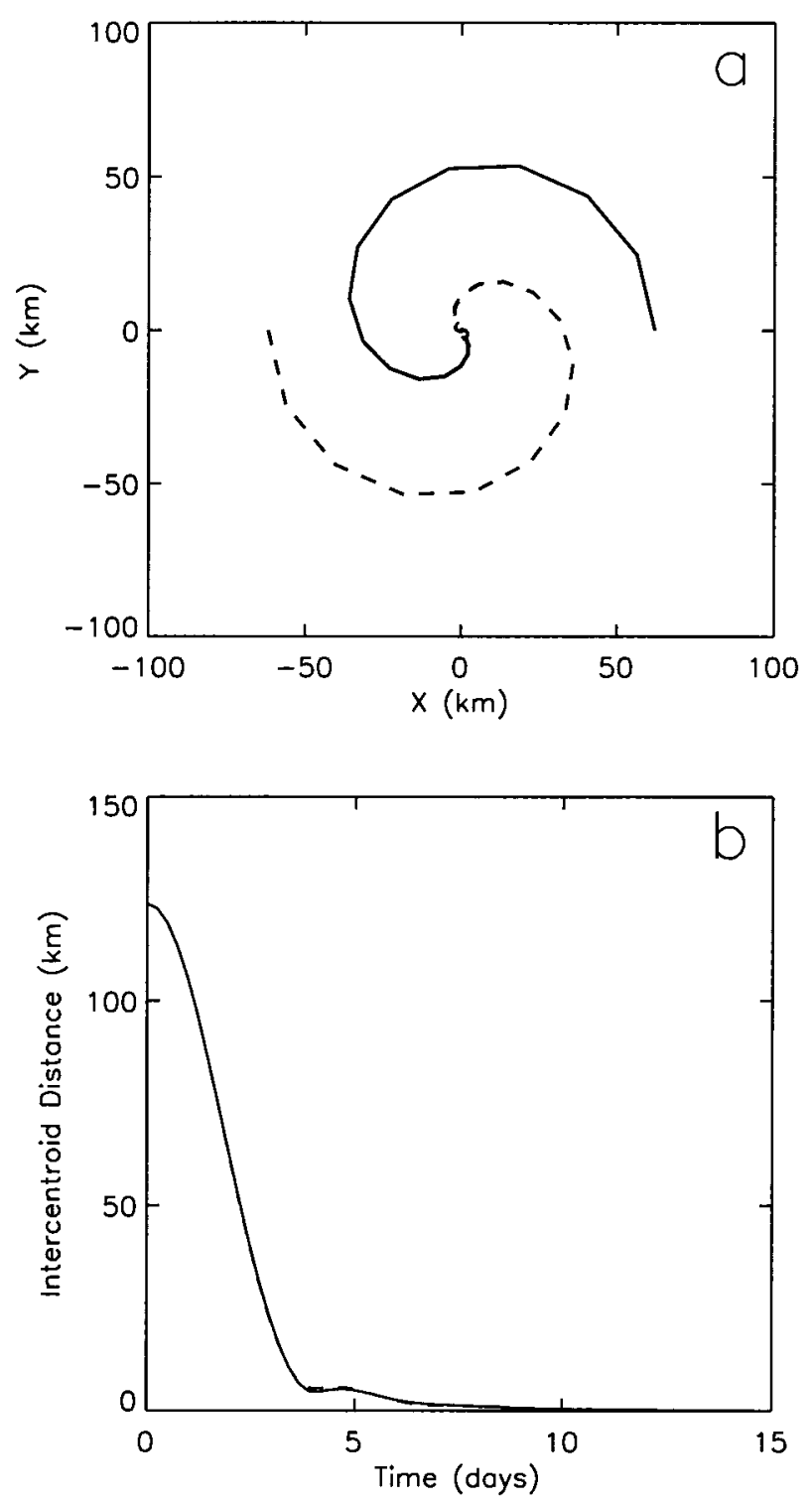

FIG. 11. As in Fig. 7 but for $\gamma_{1}=20.0 \times 10^{-6} \mathrm{~m}^{-1}$.

aforementioned grouping of eigenfunctions resembles the numerically simulated solution.

If $d \bar{\zeta} / d r$ is set to zero outside some radius, $r_{a}$, there is a possibility of the vortex supporting a smooth, discrete eigenfunction since (14) is nonsingular for $r>r_{a}$. Schecter et al. (2000) demonstrated this in the nondivergent context for two-dimensional vortex monopoles and $n=2$ by taking a profile with $d \bar{\zeta} / d r$ everywhere nonzero and setting the gradient to zero outside some radius. A discrete eigenfunction was found in this case. Upon replacing the zero vorticity gradient region with a profile having small, but nonzero gradient, an eigenanalysis showed eigenfunctions similar to the discrete eigenfunction for eigenfrequencies near the discrete value, but with small singular spikes, as in Fig. 15. The superposition of these weakly singular eigenfunctions

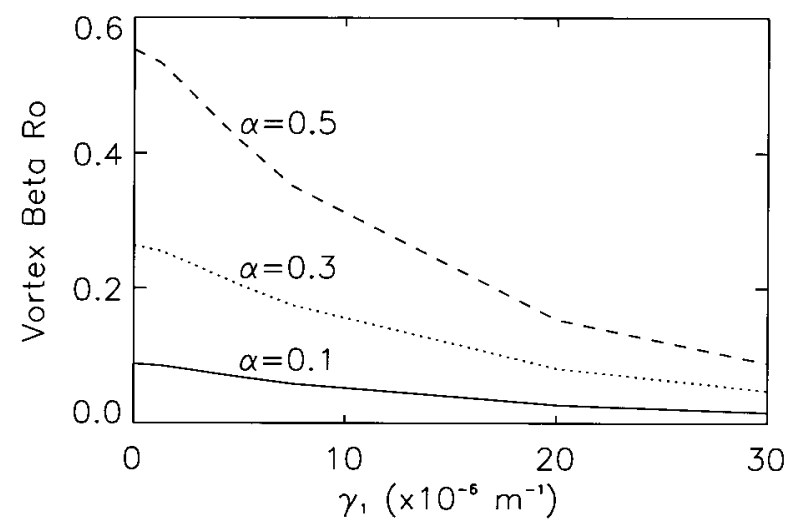

FIG. 12. Dependence of vortex beta Rossby number at $z=0$ on tilt $(\alpha)$ and $\gamma_{1}$. For consistency only $(m, n)=(1,1)$ is used to define the perturbation. The simulation for $\alpha \approx 0.5$ does contain a small barotropic wavenumber 2 component that will elevate $R_{\beta}$ slightly for all $\gamma_{1}$.

with nearly identical phase speeds is referred to as a quasi mode because of the tendency for the eigenfunctions to interfere yet still maintain a near-discrete structure in the presence of shear. Previous studies have investigated quasi modes in the upper-tropospheric flow (Rivest and Farrell 1992) and in two-dimensional nondivergent vortex flows (Schecter et al. 2000 and references therein). The singular eigenfunctions that compose the quasi mode move with slightly different phase speeds, so the quasi mode slowly decays in time as observed. From the current perspective of linear vorticity dynamics, we view this decay as occurring through the "leaking" of energy via the radial propagation of sheared vortex Rossby waves. Corotation of a geostrophic vortex with small tilt is therefore more accurately explained as the azimuthal propagation of a threedimensional quasi mode.

The longevity of the quasi mode can be assessed by considering its spectral distribution. To illustrate this, recall that in the linear approximation an arbitrary PV perturbation can be expanded in a weighted sum of the PV eigenfunctions,

$$
\hat{q}(r, t)=\sum_{k} A_{k} e^{i \omega_{k} t} \hat{\xi}_{k}(r),
$$

where $A_{k}$ is the expansion coefficient for the $k$ th PV eigenfunction, $\hat{\xi}_{k}(r)$, and $\omega_{k}$ is the corresponding eigenfrequency. The right-hand side of Eq. (15) is the discrete equivalent of a Fourier transform from the frequency domain to the time domain. For the PV perturbation given by Eq. (9), inversion of (15) at $t=0$ yields the $A_{k}$. The expansion coefficients are plotted as a function of eigenfrequency in Fig. 16 for various values of $\gamma_{1}$. A discrete mode would be represented here as a $\delta$ function in eigenfrequency space. The quasi mode is clearly identified at small $\gamma_{1}$ as the narrow spike in the expansion coefficient spectrum. As $\gamma_{1}$ increases, the quasimode spectral peak broadens and takes on a more dipolar structure. The broader the peak, and therefore the 

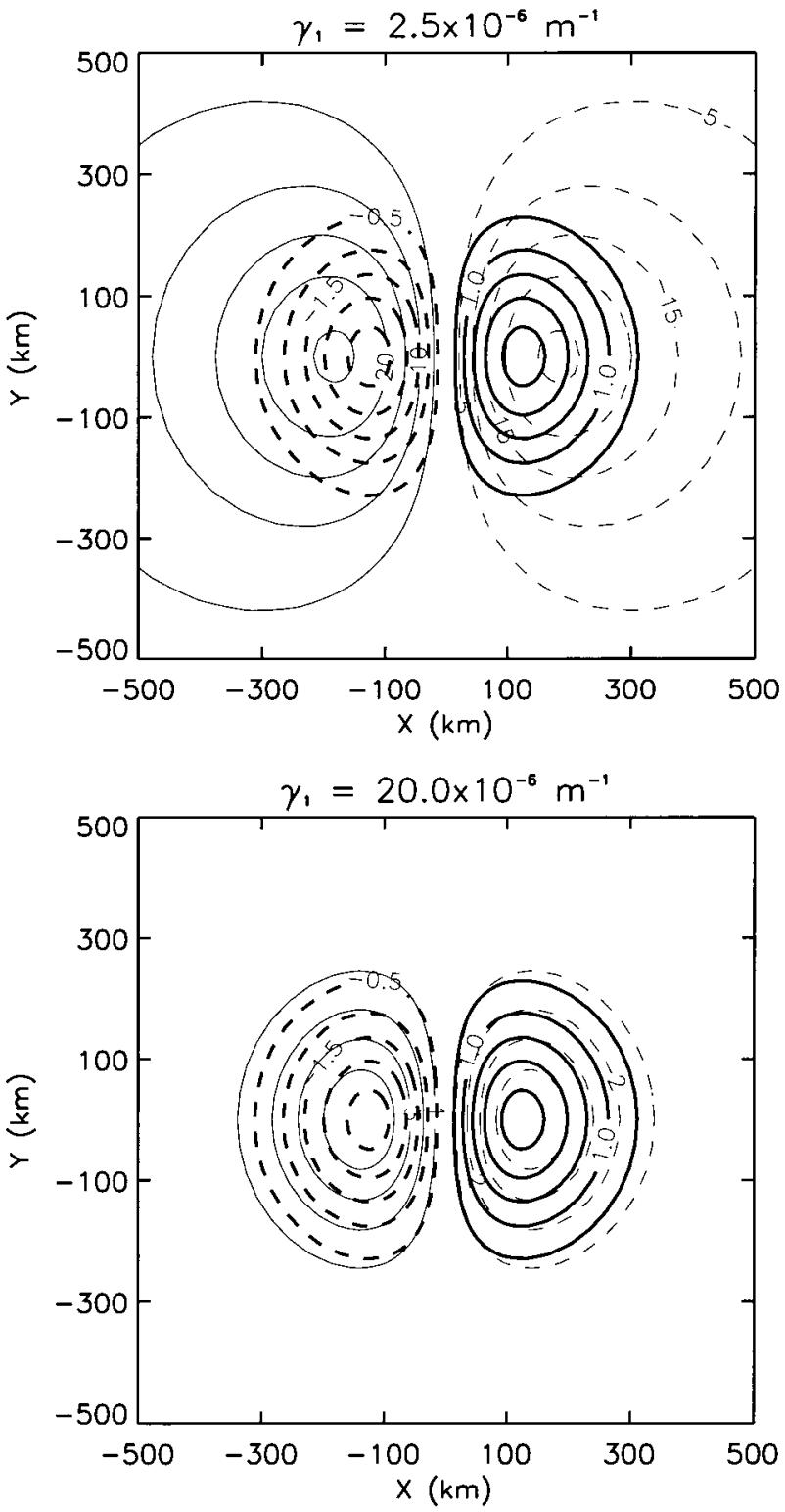

FIG. 13. Comparison of the initial perturbation PV (heavy) and streamfunction (light) for the benchmark run. Negative values are dashed. (a) $\gamma_{1}=2.5 \times 10^{-6} \mathrm{~m}^{-1}$ (b) $\gamma_{1}=20.0 \times 10^{-6} \mathrm{~m}^{-1}$. The perturbation $\mathrm{PV}$ isolines and streamlines become more parallel with increasing $\gamma_{1}$. The PV contour interval is $0.5 \times 10^{-5} \mathrm{~s}^{-1}$. Streamfunction contour interval is $5 \times 10^{4} \mathrm{~m}^{2} \mathrm{~s}^{-1}$ in (a) and $1 \times 10^{4} \mathrm{~m}^{2}$ $\mathrm{s}^{-1}$ in $(b)$

broader the distribution of phase speeds of the individual eigenfunctions that make up the quasi mode, the faster a given initial wavenumber 1 asymmetry will find itself in the continuous spectrum of sheared vortex Rossby waves. In nondivergent analyses of two-dimensional vortex monopoles, Schecter et al. (2000) were able to relate the decay rate of the quasi mode to the half-width of the spectral peak in the special case where only a single narrow peak exists. The presence of the dipole structure in the expansion coefficient spectrum found

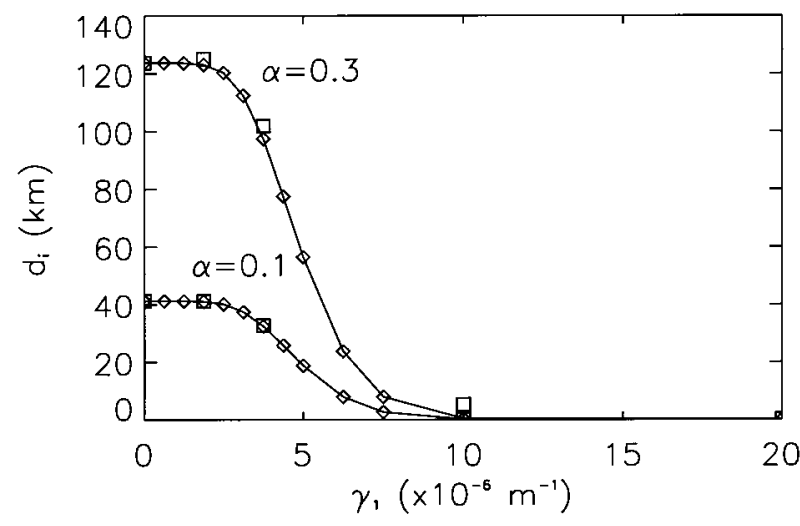

FIG. 14. The PV intercentroid separation between $z=0$ and $\mathrm{H}$ after $5 \tau_{e}$ as a function of $\gamma_{1}$. The diamonds represent linear EQB simulations and the squares nonlinear EQB simulations.

here (for $\gamma_{1} \geq 2.5 \times 10^{-6} \mathrm{~m}^{-1}$ ) prohibits an identification with a single decay rate.

According to these results we see that for a vortex monopole with small but finite-amplitude tilt and nonzero mean vorticity gradient at all radii (excluding the origin and $r=\infty$ ), alignment technically will always occur as $t \rightarrow \infty$, regardless of the nonzero value of $\gamma_{1}$. But for application to the atmosphere on meteorologically relevant timescales of a few $\tau_{e}$, it is useful and insightful to make the distinction between the quasi mode and the rest of the singular continuum of eigenfunctions, and therefore corotation and alignment.

\section{Application to tropical cyclones}

a. Vortex alignment during TC genesis

A small fraction of all tropical disturbances develop into TCs. For example, on average 100 tropical disturbances are observed in the Atlantic during hurricane season, yet only about 10-15 achieve tropical storm status (Frank 1975). In addition to the existence of lowto midlevel cyclonic vorticity, environmental factors like weak vertical shear and warm sea surface temperatures are generally regarded as necessary conditions for development. The role of vertical shear in inhibiting TC genesis and development has been explored both in observational studies (Gray 1968; Zehr 1992) and in numerical and theoretical works (Jones 1995; DeMaria 1996; Bender 1997; Frank and Ritchie 1999). According to Zehr (1992) the 200-850 mb vertical shear threshold above which development is severely inhibited is $12.5-$ $15 \mathrm{~m} \mathrm{~s}^{-1}$.

Before the underlying mechanisms for the inhibition of TC genesis by vertical shear can be understood, the unforced vortex dynamics must first be examined. We begin with the conceptual framework for TC genesis laid out by ME98. There, convectively generated lowlevel positive PV merges within an incipient midlevel vortex (e.g., mesoscale convective vortex), strength- 


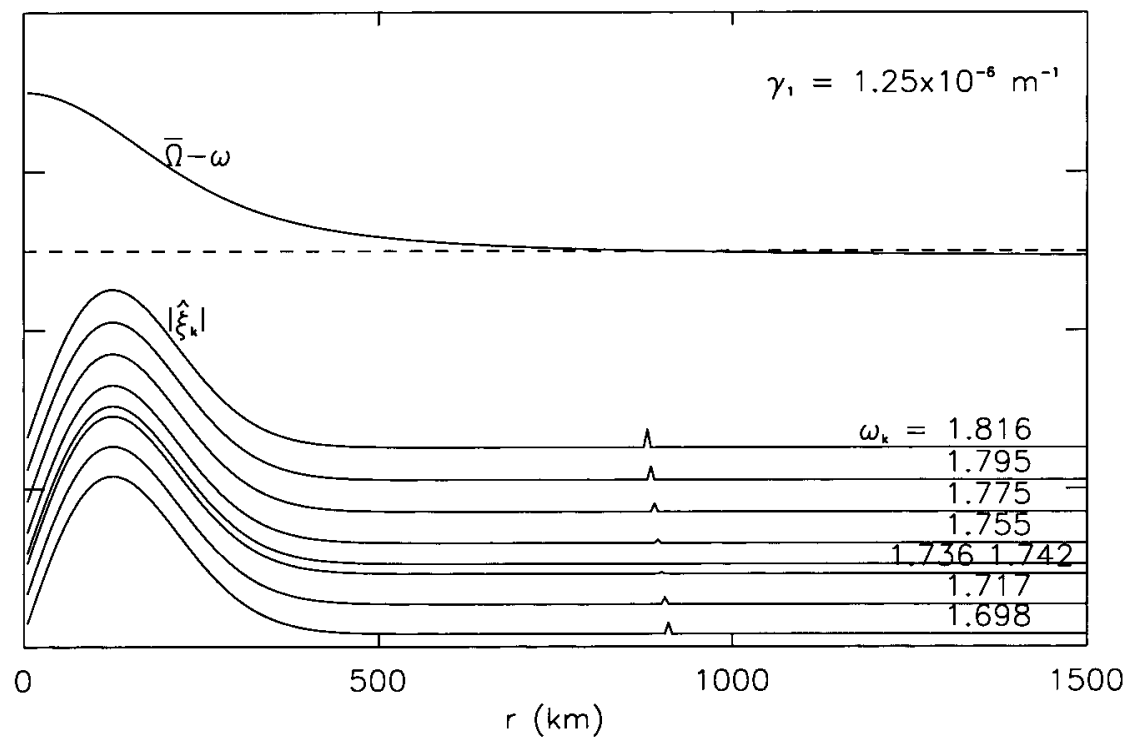

FIG. 15. The PV eigenfunction solutions to Eq. (14) for the benchmark mean vortex and ( $m$, $n)=(1,1)$. Eigenfrequencies centered on the numerically simulated rotation frequency are shown as well as $\bar{\Omega}-\omega$ for the central frequency. The units of $\omega$ are $10^{-6} \mathrm{~s}^{-1}$.

ening the cyclonic circulation beneath the midlevel vortex and initiating warm-core development. The merger of the low-level PV and midlevel vortex is similar to the vortex alignment described in section 3 , although the isolated nature of the convectively generated PV makes for a less vertically coherent vortex structure through the lower to middle troposphere. Nevertheless, the linear alignment ideas discussed in section 3 can be applied to the nonaxisymmetric genesis model of ME98 to elucidate the underlying dynamical mechanisms. ME98 suggested the role of vortex Rossby waves in the merger process by showing good agreement between nonlinear and quasi-linear predictions of the mean flow change resulting from the interaction of a barotropic wavenumber 2 asymmetry with a barotropic mean vor-

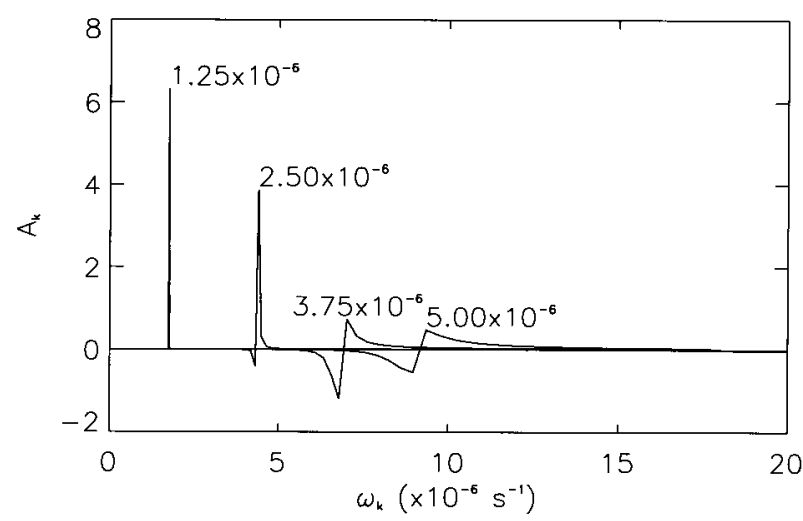

FIG. 16. Expansion coefficient $A_{k}$ as a function of eigenfrequency $\omega_{k}$ for values of $\gamma_{1}$ in the quasi-mode and transition regimes. The wavenumber 1 asymmetry given by Eq. (9) and eigenfunctions shown in Fig. 15 were used to obtain $A_{k}$. tex. Quasi-linear estimates of the mean flow change for a baroclinic two-cluster PV anomaly on a barotropic vortex also agreed with nonlinear simulations. Here we explicitly compare linear and nonlinear simulations of the merger of a single-cluster convectively generated PV anomaly within a weak vortex to demonstrate the role of vortex Rossby waves. Although a midlevel vortex is a more physically relevant mean state than a barotropic vortex in the context of TC genesis, we use the latter to convey the basic dynamics of the merger process.

The same barotropic mean vortex as ME98 is used (see Fig. 1). The single-cluster isolated anomaly with positive (negative) PV at lower (upper) levels is given by

$$
q_{\mathrm{sc}}(r, \lambda, z)=\kappa \bar{q}_{0} e^{-(\beta \delta r)^{2}} \cos \left(\frac{\pi z}{H}\right),
$$

where $\kappa$ is an amplitude factor, $\beta$ is the inverse decay length of the asymmetry, and $(\delta r)^{2}=\left(x-x_{c}\right)^{2}+(y$ $\left.-y_{c}\right)^{2}$. The parameters $x_{c}$ and $y_{c}$ denote the Cartesian location of the asymmetry center. In the experiment presented here $\kappa=0.5$ and $\beta=1.0 \times 10^{-5} \mathrm{~m}^{-1}$. In order to initialize the EQB model with a barotropic mean vortex, as in the previous section, the azimuthal wavenumber zero component of the anomaly at $z=0$ is added to the preexisting mean vortex at all heights. The resulting mean PV still monotonically decreases with radius. In contrast to section 3 we do allow the generation of azimuthal-mean vertical wavenumber 2 in the nonlinear EQB model through the wave-mean interaction of $(m, n)=(1,1)$, but it is incorporated into the barotropic mean vortex (see appendix A). No modifi- 
cations to (16) were made in the QG3D model, so the mean vortex contains a small baroclinic component. The grid spacing for the QG3D model simulation is $7.5 \mathrm{~km}$ and the domain is now $1500 \mathrm{~km} \times 1500 \mathrm{~km}$. Horizontal "del-squared" diffusion of PV has also been included with $\nu=100 \mathrm{~m}^{2} \mathrm{~s}^{-1}$.

The isolated anomaly is placed inside the RMW of the preexisting vortex at $x_{c}=125 \mathrm{~km}$ and $y_{c}=0 \mathrm{~km}$ to simulate an outbreak of convection near the vortex core. Figure 17 shows the PV evolution over $2.5 \tau_{e}$ simulated by the QG3D and linear EQB models for $\gamma_{1}=$ $3.14 \times 10^{-6} \mathrm{~m}^{-1}$. Although we are now considering tropical conditions, a value of $f_{0}=1.0 \times 10^{-4} \mathrm{~s}^{-1}$ was used as a crude way of including the vorticity of the vortex in the definition of $\gamma_{1}$ (see ME98). The linear and nonlinear simulations agree well, consistent with small $R_{\beta}(\sim 0.2)$. Because of the greater departure of the horizontal structure of the initial PV asymmetry from the barotropic pseudomode, more sheared vortex Rossby wave dispersion is evident than in the tilted vortex experiments. At longer times the sheared waves symmetrize, leaving just the quasi mode, which appears to frustrate the complete merger of the isolated PV anomaly within the incipient vortex.

While complete merger is not expected during the time period considered here due to the presence of the quasi mode, forward Lagrangian trajectories from the linear simulation do show that PV from the low-level positive anomaly is transported radially inward into the vortex core (see Fig. 18). Of course total PV is not materially conserved as parcels move toward the vortex center in the linear approximation, as evidenced by the reduction in peak PV in Fig. 17. Nevertheless, the linear vortex Rossby waves do irreversibly transport PV. The radially inward transport of cyclonic eddy vorticity and eddy PV implies a strengthening of the low-level mean tangential winds in the vortex core by Stokes's theorem, and vice versa. This is illustrated in Fig. 19 for the above experiment. Over $2.5 \tau_{e}$ the QG3D model shows an increase in low-level tangential winds of approximately $0.21 \mathrm{~m} \mathrm{~s}^{-1}$ radially inside the location of the initial PV asymmetry. The nonlinear EQB simulation produces a similar structure for the tangential wind change, $\delta \bar{v}$, but with a maximum value of $0.18 \mathrm{~m} \mathrm{~s}^{-1}$.

It is not surprising that the QG3D and nonlinear EQB models should agree as well as they do given that the low-level PV evolution is largely captured by linear vortex Rossby wave dynamics. Since wave-wave interactions are small (recall $R_{\beta} \sim 0.2$ ), the bulk of the mean flow change is effected by the self-interaction of vertical wavenumber 1 (i.e., wave-mean interaction) as shown in Fig. 19. Montgomery and Kallenbach predicted, and it was later confirmed in a nondivergent model for small but finite-amplitude disturbances (ME98) and for near order 1 amplitude disturbances (Enagonio and Montgomery 2001), that in such a situation one can use the linear solution to estimate the mean flow change that would occur in a model where the wave-mean interaction was computed explicitly. The so-called quasi-linear approximation is an estimate because the mean flow is prohibited from changing in the linear model. The feedback on the wave dynamics is not captured as it is in the wave-mean model.

The quasi-linear formulation in the QG context is obtained following Held and Phillips (1987) and Holton (1992, section 12.4). The change in mean PV is given by

$$
\delta \bar{q}=\frac{1}{r} \frac{\partial}{\partial r}(\delta A),
$$

where $A(r, z, t) \equiv r \overline{q^{\prime 2}} /(2 d \bar{q} / d r)$ is the wave activity (or pseudo angular momentum) and $\delta$ denotes the difference between a mean quantity at $t \rightarrow \infty$ and $t=0$.

Given the PV perturbation,

$$
q^{\prime}=q_{1}(r, \lambda, t) \cos \left(\frac{\pi z}{H}\right)
$$

the wave activity can be written as

$$
A=\frac{\tilde{A}}{2}\left[1+\cos \left(\frac{2 \pi z}{H}\right)\right],
$$

where $\tilde{A}=r \overline{q_{1}^{2}} /(2 d \bar{q} / d r)$. Therefore $\delta A$ has both a barotropic and vertical wavenumber 2 component. The mean angular momentum change due to the barotropic component is obtained directly as

$$
\delta\left(r \bar{v}_{0}\right)=\frac{1}{2} \delta \tilde{A},
$$

while the wavenumber 2 component is obtained by first solving the invertibility relation,

$$
\frac{1}{r} \frac{\partial}{\partial r}\left(r \frac{\partial}{\partial r}\left(\delta \bar{\psi}_{2}\right)\right)-\gamma_{2}^{2} \delta \bar{\psi}_{2}=\frac{1}{r} \frac{\partial}{\partial r}\left(\frac{\delta \tilde{A}}{2}\right),
$$

and then differentiating the incremental streamfunction

$$
\delta \bar{v}_{2}=\frac{\partial}{\partial r}\left(\delta \bar{\psi}_{2}\right) .
$$

The total mean angular momentum change is the sum of these two contributions:

$$
\delta(r \bar{v})=\delta\left(r \bar{v}_{0}\right)+\delta\left(r \bar{v}_{2}\right) \cos \left(\frac{2 \pi z}{H}\right) .
$$

Since $\gamma_{2}$ is relatively small in our case, we may assume $\delta \bar{v}_{2}=\delta \bar{v}_{0}$ to provide an upper bound on $\delta \bar{v}$. The quasilinear result derived from the linear EQB simulation is shown in Fig. 19. It not only replicates the general radial structure of the nonlinear $\delta \bar{v}$ predictions, but is also of the correct magnitude.

\section{b. Alignment of vertically coherent TCs}

Once the developing TC becomes vertically coherent, the linear alignment model can be directly applied to determine whether or not the vortex will remain aligned. 

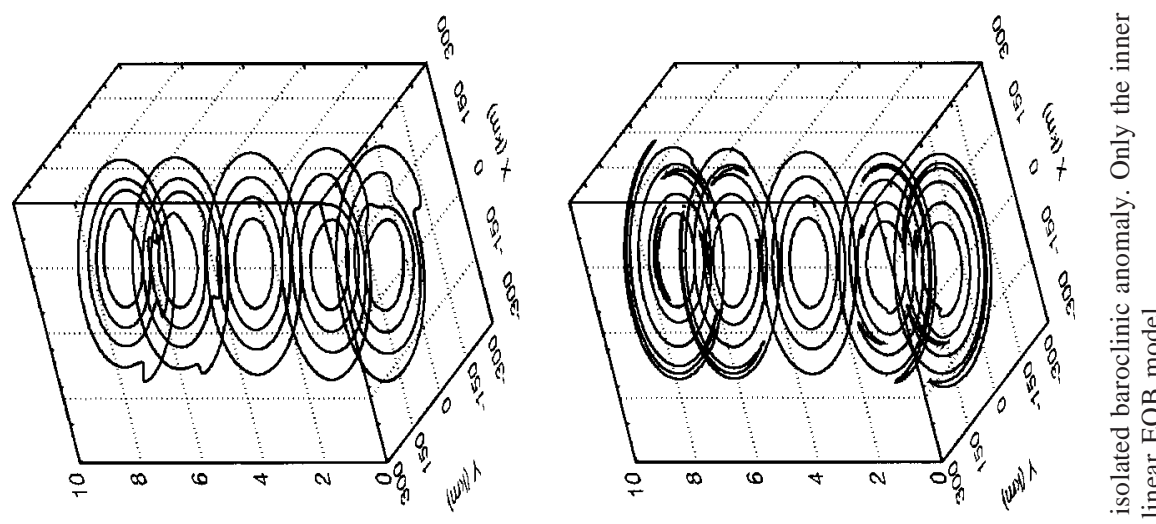

을
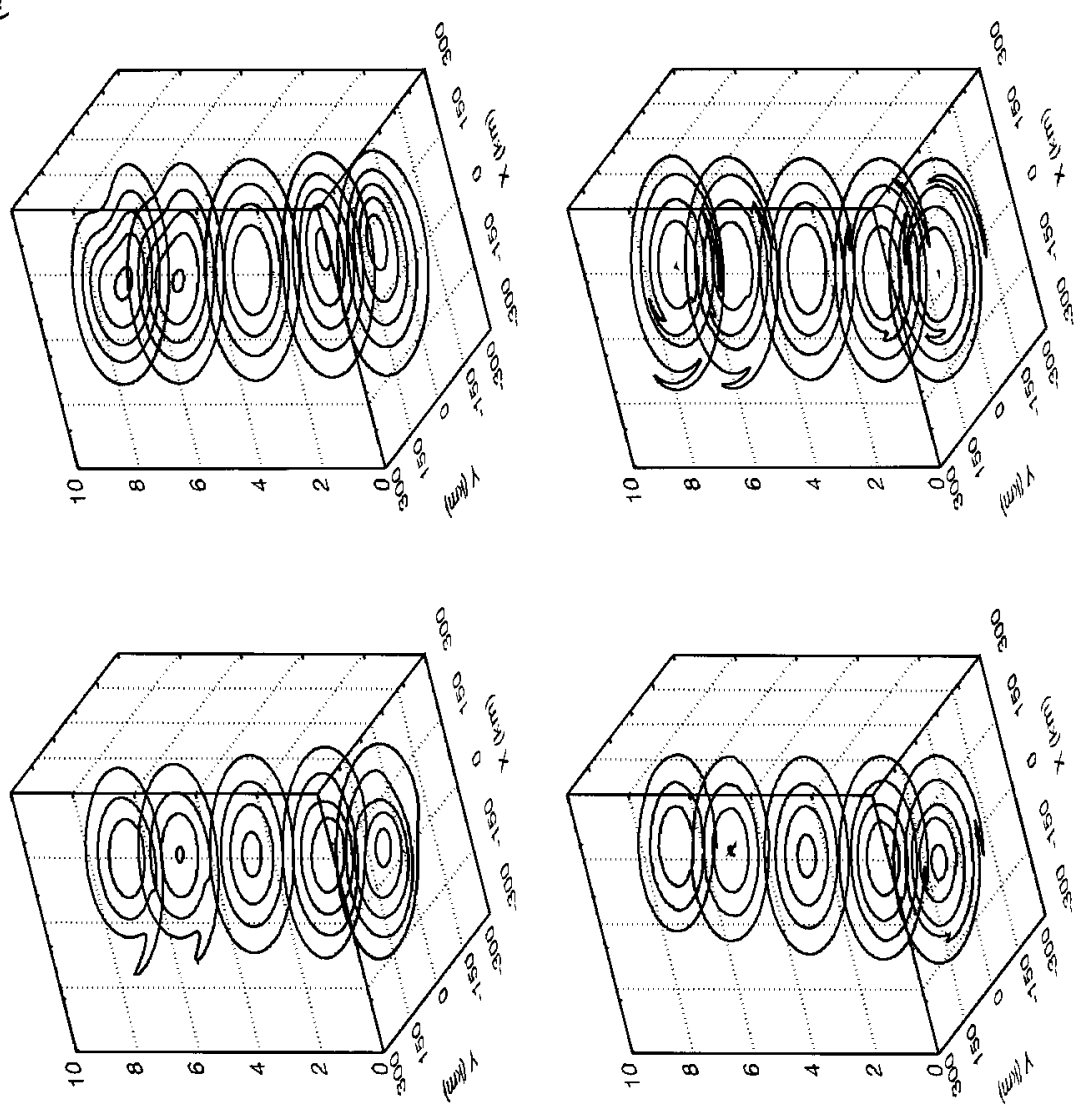

ธิธ
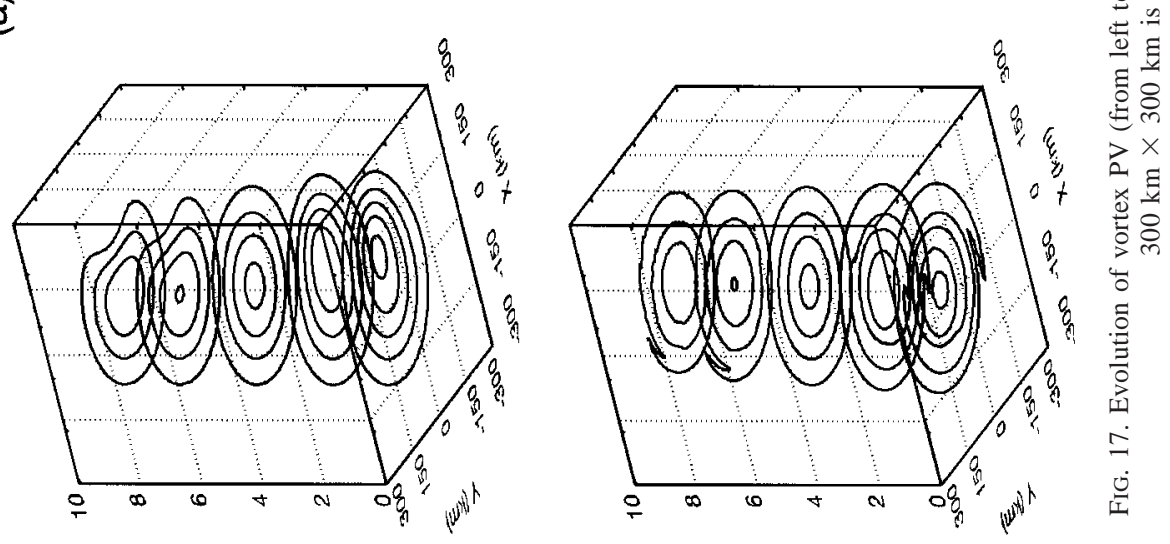


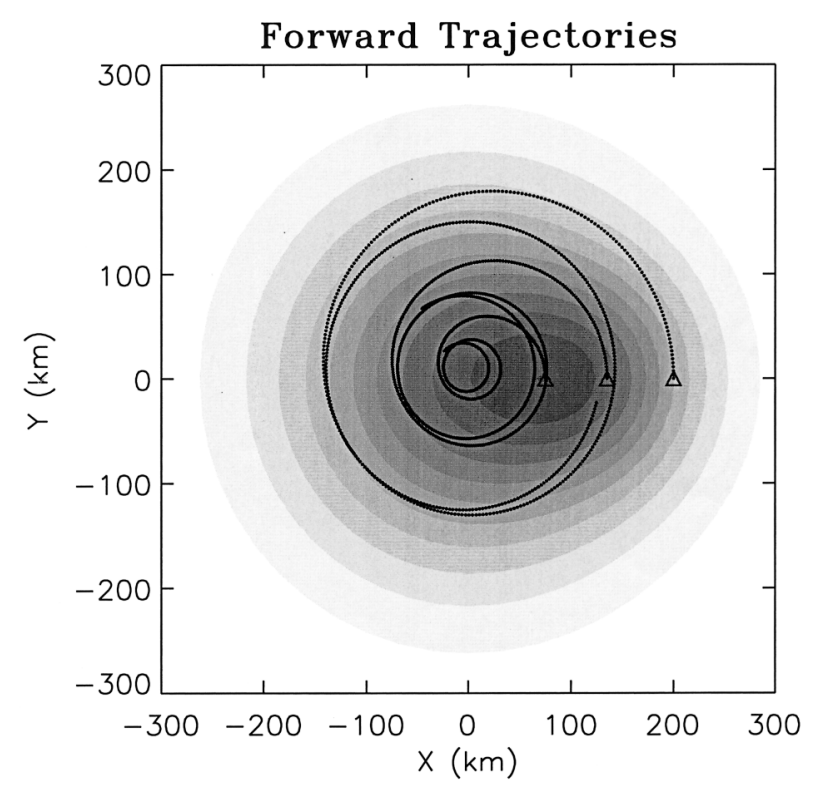

FIG. 18. Forward Lagrangian trajectories of parcels originating within the isolated anomaly at $z=0$ computed using winds simulated by the linear EQB model over $2.5 \tau_{e}$. Note that high PV is transported in toward the vortex center.

One can interpret the tilted vortex of section 3 as having resulted from some external forcing, such as environmental vertical shear or horizontal strain associated with nearby vortices. Depending on the horizontal scale of the vortex and $\gamma_{1}$, the vortex will either return to a vertically aligned state through the sheared vortex Rossby wave mechanism or precess about its midlevel centroid due to the wavenumber 1 quasi-mode propagation.
For tropical conditions $f_{0}=5 \times 10^{-5} \mathrm{~s}^{-1}, H=15 \mathrm{~km}$, and $N=10^{-2} \mathrm{~s}^{-1}$, resulting in $\gamma_{1}=1.0 \times 10^{-6} \mathrm{~m}^{-1}$. For the mean vortex depicted in Fig. 1, $\gamma_{1}<L^{-1}$, well within the nonalignment regime shown in Fig. 14. In the nonaligned state the vortex is especially vulnerable to further external forcing.

Recently Dritschel and de la Torre Juárez (1996), using a linear stability analysis and a multilayer QG CD model, found that a vortex column subjected to twodimensional strain will become unstable and reduce its vertical scale if the ratio of vortex height to width is greater than about $3 f / N$, or equivalently $\gamma_{1}<\pi / 6 L$. For the benchmark vortex with $L=200 \mathrm{~km}$, the vortex breakdown criterion is $\gamma_{1}<2.5 \times 10^{-6} \mathrm{~m}^{-1}$. Recall that this range of $\gamma_{1}$ was identified using linear theory in section 3 as the nonalignment regime. Therefore, a weak tropical vortex supporting a quasi mode will tend to reduce its vertical scale in the presence of external shear until the alignment regime is reached.

In the case of TCs, the asymmetric transverse circulation required to maintain thermal wind balance in the small- $\gamma_{1}$ tilted configuration may actually help development. If the persistent asymmetric low-level convergence and enhanced convection in the down-tilt quadrant of the storm (Jones 1995; Reasor et al. 2000) is larger than would be produced through symmetric mechanisms alone, an enhanced strengthening of the primary vortex circulation through symmetrization of convectively generated PV may occur (MK; ME98; Möller and Montgomery 2000). According to the QG vortex alignment mechanism, even though the vortex has strengthened, it would continue to precess about its midlevel centroid since $\gamma_{1}$ has not changed. If one crude-

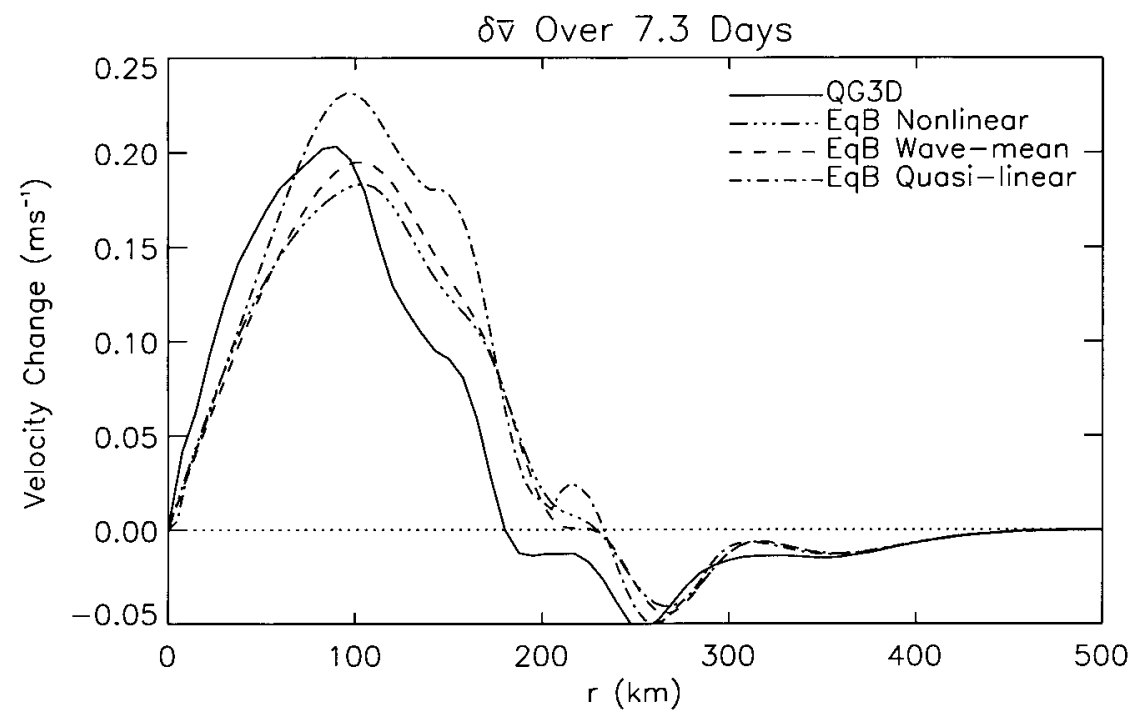

FIG. 19. Change in azimuthal-mean tangential velocity at $z=0$ over $2.5 \tau_{e}$ as a function of radius for the isolated anomaly experiment. Shown are results from the QG3D and EQB models illustrating the quasi-linear nature of the low-level intensification of the mean flow by vortex Rossby waves. 
ly extends the findings of section 3 to include finite Rossby number effects by replacing $f_{0}^{2}$ in the expression for $\gamma_{1}^{2}$ with the product of the modified Coriolis parameter and absolute vorticity of the associated vortex flow, the increase in vortex strength will increase $\gamma_{1}$. The vortex could potentially leave the non-alignment regime and realign itself without changing its vertical scale. Preliminary experiments using the asymmetric balance (AB) model (Shapiro and Montgomery 1993) in which the standard Rossby number approaches unity show good agreement with the QG results of section 3 (Reasor 2000). More recent results using a linear primitive equation model suggest that alignment of a tilted vortex initially in a corotation regime can indeed occur by increasing the vortex strength. Further results regarding the alignment of rapidly rotating vortices will be reported upon in a forthcoming publication.

\section{Summary and discussion}

The evolution of an initially tilted PV column for which the nonlinear advective PV tendency is small compared to the linear radial advective tendency (i.e., $R_{\beta} \ll 1$ ) is captured by linear vortex Rossby wave processes. This condition is generally met for vortices with overlapping upper- and lower-level PV cores. In this situation the vortex is meaningfully decomposed into azimuthal-mean and perturbation components. Numerical vortex simulations based on the linear equivalent barotropic QG system, and validated with a nonlinear three-dimensional QG model, show a dependence on the internal Rossby deformation radius consistent with previous studies. The physical explanation for the tilted vortex evolution, however, departs from these studies.

In the nondivergent limit (i.e., infinite internal deformation radius) the stationary pseudomode is recovered (MK). For large but finite internal deformation radii the upper and lower PV anomalies slowly corotate. As the deformation radius is decreased, the corotation frequency increases. An azimuthally propagating quasi mode with the vertical structure of the first internal baroclinic mode and wavenumber 1 azimuthal structure is responsible for the corotation and inhibits vortex alignment on meteorologically pertinent timescales. The quasi mode, defined here as a superposition of weakly singular eigenfunctions sharply peaked in the phase speed spectrum, decays slowly in the presence of differential rotation due to radial energy propagation by sheared vortex Rossby waves. A transition region centered near the horizontal vortex scale separates this regime from the alignment regime at smaller internal deformation radii. The transition is accounted for by the spectral broadening of the quasi mode. In the alignment regime the quasi mode is not present and initial perturbations project primarily onto the (non-quasi-modal) continuous spectrum of vortex Rossby waves whose integrated perturbation energy decays algebraically to zero in the limit of long times. Alignment is defined here as when the intercentroid separation distance between upper- and lower-level PV anomalies is zero. We believe this to be a more accurate definition than used by Polvani (1991) who defined alignment as a net decrease in the intercentroid separation distance over one precession period of the vortex. In the single-interface CD model, Rossby edge waves persist where complete alignment is observed for broad, continuously distributed vortices. Sheared linear vortex Rossby waves promote the irreversible redistribution of PV necessary for complete alignment.

We argued that linear theory captures the essence of corotation even as $R_{\beta}$ approaches unity due to the robustness of the quasi mode. In this regime nonlinear advection simply tries to counteract the sheared vortex Rossby wave dispersion. As the internal deformation radius is decreased within the alignment regime, $R_{\beta}$ for the baroclinic portion of the asymmetry decreases rapidly. Thus, linear theory will tend to capture the vortex alignment process for a wider range of initial tilts the smaller the internal deformation radius. The largest tilt considered here was when the RMW of the upper-level vortex overlapped the vortex center at lower levels. For an internal deformation radius near $50 \mathrm{~km}$ we estimate that $R_{\beta}$ is less than 0.2 for this tilted configuration. It is possible that alignment can still be approximately described with linear dynamics even for vortices with barely overlapping upper- and lower-level PV cores.

These ideas are believed to have practical application to the problem of tropical cyclogenesis. One of the basic questions in TC research is how a weak vortex with nearby convection resists the effects of external shear and strengthens. The Rossby wave interpretation of the asymmetric TC genesis mechanism of ME98 was further clarified here by explicitly demonstrating that the merger and alignment of small but finite-amplitude convectively generated low-level positive PV within the RMW of a preexisting vortex is captured by linear vortex Rossby wave processes. The attendant strengthening of the low-level mean vortex was also captured by wave-mean dynamics. In typical tropical conditions this process is frustrated by vertical shear. Smith et al. (2000) found that a tilted TC-like vortex will either break apart under the influence of vertical shear or corotate. Depending on the value of the internal deformation radius, the linear alignment mechanism discussed here suggests that the tilting of a vertically aligned vortex by shear will either project perturbation energy primarily onto the wavenumber 1 quasi mode or entirely onto sheared vortex Rossby waves. In the latter case axisymmetrization of the perturbation PV through the interaction of vortex Rossby waves with the mean flow will tend to resist the tilting by shear. In this regard, vertical shear acts as a sheared vortex Rossby wave generator. This interpretation of the interaction of a vortex with vertical shear is another way of viewing the vortex tilt evolution described by Jones (1995) for $R_{\beta}<1$.

In addition to adding vertical shear to the linear problem, the next step is to extend the QG results to more 
rapidly rotating flows. The vertical penetration depth will not only become a function of radius, but will also increase over the QG value. This was clearly demonstrated by Shapiro and Montgomery (1993) in the context of the $\mathrm{AB}$ formulation for hurricane-like flows, where the local penetration depth is proportional to the square root of the product of the modified Coriolis parameter and absolute vorticity of the associated vortex flow. Preliminary simulations in which the linearized equivalent barotropic $\mathrm{AB}$ model is initialized with our tilted benchmark vortex agree with the corresponding QG simulations (Reasor 2000). Further work is required to understand the effect of variable penetration depth on the evolution of stronger tilted vortices. It is possible that a quasi mode in quasi-gradient balance exists at higher swirl speeds. Its characteristics and relevance to hurricane-like flows (e.g., track wobbles) would be of great interest. This will be the subject of future investigation.

Acknowledgments. This work was supported by National Science Foundation Grant ATM-9732678 and the Cooperative Institute for Research in the Atmosphere (CIRA) at Colorado State University, and represents part of the first author's Ph.D. thesis at CSU. We thank three anonymous reviewers for helping to improve the clarity of the manuscript. We also thank Dr. Janice Enagonio for her help with the three-dimensional QG model and for implementation of the Lagrangian trajectory code. Finally we thank Mr. John Persing for helping with the creation of the three-dimensional volume visualizations presented here.

\section{APPENDIX A}

\section{Nonlinear QG Equivalent Barotropic Model}

For the tilted vortex (section 3 ) and $m=1$ isolated anomaly (section 4) experiments, it is sufficient to simulate the vortex evolution using only the barotropic mode $(m=0)$ and first internal baroclinic mode $(m=$ 1). Truncating (3) at $m=1$ and then substituting into the PV equation (1) yields the following nonlinear PV tendency equations for $m=0$ and 1 , respectively:

$$
\begin{aligned}
& \left(\frac{\partial}{\partial t}+\frac{1}{r} \frac{\partial \psi_{0}}{\partial r} \frac{\partial}{\partial \lambda}-\frac{1}{r} \frac{\partial \psi_{0}}{\partial \lambda} \frac{\partial}{\partial r}\right) q_{0}(r, \lambda, t) \\
& +\frac{1}{2}\left(\frac{1}{r} \frac{\partial \psi_{1}}{\partial r} \frac{\partial}{\partial \lambda}-\frac{1}{r} \frac{\partial \psi_{1}}{\partial \lambda} \frac{\partial}{\partial r}\right) q_{1}(r, \lambda, t)=0
\end{aligned}
$$

and

$$
\begin{aligned}
\left(\frac{\partial}{\partial t}\right. & \left.+\frac{1}{r} \frac{\partial \psi_{0}}{\partial r} \frac{\partial}{\partial \lambda}-\frac{1}{r} \frac{\partial \psi_{0}}{\partial \lambda} \frac{\partial}{\partial r}\right) q_{1}(r, \lambda, t) \\
& +\left(\frac{1}{r} \frac{\partial \psi_{1}}{\partial r} \frac{\partial}{\partial \lambda}-\frac{1}{r} \frac{\partial \psi_{1}}{\partial \lambda} \frac{\partial}{\partial r}\right) q_{0}(r, \lambda, t)=0
\end{aligned}
$$

This system of equations will tend to underestimate the magnitude of the azimuthal mean flow change since the $m=2$ tendency associated with the self-interaction of $(m, n)=(1, n)$ is excluded.

In section 3, where the wave-mean interaction is not critical to understanding the vortex evolution, the above truncated system is solved. The ()$_{m}$ are first expanded in a truncated azimuthal Fourier series following appendix B of ME98:

$$
\begin{aligned}
& \psi_{m}(r, \lambda, t)=\sum_{n=-N}^{N} \hat{\psi}_{m n}(r, t) e^{i n \lambda} \text { and } \\
& q_{m}(r, \lambda, t)=\sum_{n=-N}^{N} \hat{q}_{m n}(r, t) e^{i n \lambda},
\end{aligned}
$$

where $n$ is the azimuthal wavenumber and $N$ is the azimuthal mode truncation. This semispectral formulation is advantageous for swirling flows where the mean flow dominates the asymmetric component of the flow. Because the radial and azimuthal resolutions are independent, one can choose the minimum azimuthal resolution needed to simulate the vortex evolution, and thus reduce computational time. Substitution of (A3) and (A4) into the flux form of Eqs. (A1)and (A2) yields, respectively,

$$
\begin{aligned}
& \frac{\partial \hat{\psi}_{0 n}}{\partial t}=\nabla_{0 n}^{-2} \hat{F}_{0 n}(r, t) \quad \text { and } \\
& \frac{\partial \hat{\psi}_{1 n}}{\partial t}=\nabla_{1 n}^{-2} \hat{F}_{1 n}(r, t)
\end{aligned}
$$

where

$$
\begin{aligned}
\hat{F}_{0 n}(r, t)= & \frac{1}{r}\left\{\sum_{\substack{|k| \leq N \\
|n-k| \leq N}}\left[i k \frac{\partial}{\partial r}\left(\hat{\psi}_{0, k} \hat{q}_{0, n-k}\right)-i n \hat{q}_{0, n-k} \frac{\partial \hat{\psi}_{0, k}}{\partial r}+\frac{1}{2}\left(i k \frac{\partial}{\partial r}\left(\hat{\psi}_{1, k} \hat{q}_{1, n-k}\right)-i n \hat{q}_{1, n-k} \frac{\partial \hat{\psi}_{1, k}}{\partial r}\right)\right]+i n \frac{d \bar{\zeta}}{d r} \hat{\psi}_{0, n}-i n \frac{\partial \bar{\psi}}{d r} \hat{q}_{0, n}\right\} \\
& +\nu \nabla_{0 n}^{2} \hat{q}_{0, n}, \\
\hat{F}_{1 n}(r, t)= & \frac{1}{r}\left\{\sum_{\substack{|k| \leq N \\
|n-k| \leq N}}\left[i k \frac{\partial}{\partial r}\left(\hat{\psi}_{0, k} \hat{q}_{1, n-k}\right)-i n \hat{q}_{1, n-k} \frac{\partial \hat{\psi}_{0, k}}{\partial r}+\left(i k \frac{\partial}{\partial r}\left(\hat{\psi}_{1, k} \hat{q}_{0, n-k}\right)-i n \hat{q}_{0, n-k} \frac{\partial \hat{\psi}_{1, k}}{\partial r}\right)\right]+i n \frac{d \bar{\zeta}}{d r} \hat{\psi}_{1, n}-i n \frac{\partial \bar{\psi}}{d r} \hat{q}_{1, n}\right\} \\
& +\nu \nabla_{0 n}^{2} \hat{q}_{1, n},
\end{aligned}
$$




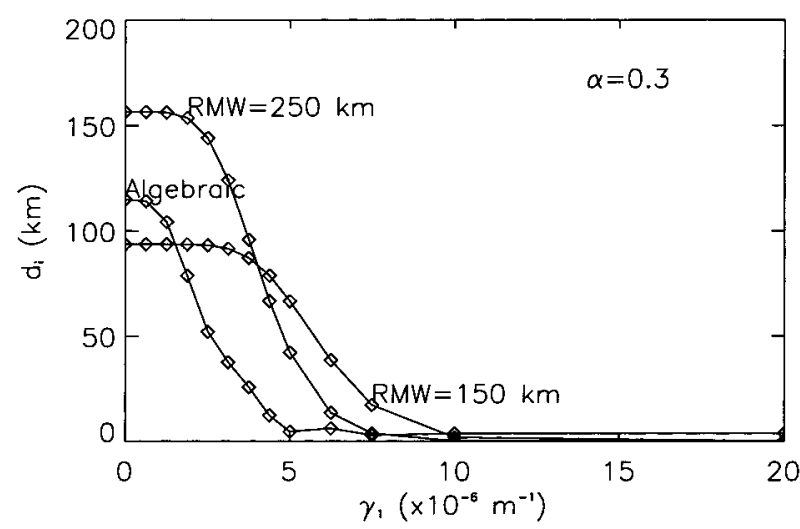

FIG. B1. As in Fig. $14(\alpha=0.3)$, but for Gaussian vortices smaller and larger than the benchmark vortex. Also shown are results for a vortex having an algebraic dependence of PV on radius given by Eq. (B1). The RMW in the latter case is $200 \mathrm{~km}$.

and $\nabla_{m n}^{2}=(1 / r) \partial / \partial r+\partial^{2} / \partial r^{2}-n^{2} / r^{2}-\gamma_{m}^{2}$. The last term in both equations is the explicit diffusion. Note that the linear terms involve only the barotropic component of the azimuthal mean vortex, that is, $\psi(r, t)=$ $\hat{\psi}_{00}(r, t)$ and $\bar{q}(r, t)=\hat{q}_{00}(r, t)$. Consistent with the barotropic mean vortex constraint, we impose $\hat{F}_{10}=0$.

In section 4 where the mean flow change predicted by the QG3D and EQB models is compared, we wish to include the full effect of the self-interaction of $(m$, $n)=(1, n)$, that is, both the $(0,0)$ and $(2,0)$ contributions. The wave-mean terms that contribute to the tendency in $(0,0)$ are in fact the same terms that contribute to the tendency in $(2,0)$. Thus, to account for the $m=2$ tendency we simply double the wave-mean terms in the $(0,0)$ equation. This approximation is equivalent to assuming $m=0$ in the inversion of potential vorticity for streamfunction, and will tend to overestimate the magnitude of the mean-flow change. The inclusion of $m=2$ in this way is consistent with the quasi-linear approximation presented in section 4 .

\section{APPENDIX B}

\section{Sensitivity to Horizontal PV Profile}

Changing the width of the horizontal PV profile does not alter the basic results presented here. Figure B1 shows the PV intercentroid separation distance after $5 \tau_{e}$ as a function of $\gamma_{1}$ for mean vortices smaller and larger than that used in our benchmark simulation (see Fig. $1)$. Recall that the vortex Burger number $\left(L_{R} / L\right)^{2}$ is the parameter that appears in the nondimensionalized invertibility relation and determines the partitioning between vortical and thermal PV. We observed in section $3 \mathrm{c}$ that as the Burger number decreases from the nondivergent limit (i.e., $\gamma_{1}$ increases from zero) the vortex moves from the quasi-mode to alignment regime. For a value of $\gamma_{1}$ within the transition regime an increase (decrease) in $L$ will decrease (increase) the vortex Bur- ger number, bringing the vortex closer to (further from) the alignment regime. As Fig. B1 shows, the result is that the quasi-mode regime contracts with increasing vortex scale.

Also shown in Fig. B1 is the separation distance using a PV profile that decreases algebraically with radius:

$$
\bar{q}(r)=\frac{\bar{q}_{\max }}{\left(1+(r / R)^{2}\right)^{2}},
$$

where $R=200 \mathrm{~km}$ and $\bar{q}_{\max }=1.0 \times 10^{-4} \mathrm{~s}^{-1}$. The alignment, transition, and corotation regimes are evident, but their location in $\gamma_{1}$ space differs from that of the benchmark Gaussian vortex in spite of similar inner-core structures. The region of nonnegligible vorticity gradient associated with the algebraic profile extends to larger radius than in the benchmark Gaussian case. Thus, the scale of the vortex is larger in the former case, accounting for the more extensive alignment regime.

According to arguments presented in section $3 \mathrm{~d}$, the existence of the quasi mode depends on there being a region of near-zero mean vorticity gradient beyond some radius. As the scale of the vortex monopole increases, the radial region of small, but nonzero vorticity gradient decreases. Thus, there is a smaller range of frequencies for which Eq. (14) will support the weak singularity associated with the quasi-mode solutions. Precisely how this translates into the $\gamma_{1}$ dependence shown in Fig. B1 will depend on how vortex structure and $\gamma_{1}$ determine the quasi-mode frequency. This, we currently do not know, but hope to understand through ongoing work.

\section{APPENDIX C}

\section{Simulating Vortex Alignment: Vortex Patches versus Continuous TC-like Profiles}

Closed-form EQB solutions to the linear tilted vortex problem can be obtained for mean vortices whose radial structure is that of a vortex patch, that is,

$$
\bar{\zeta}(r)= \begin{cases}\bar{\zeta}_{\max }, & 0 \geq r<a \\ 0, & a<r \leq \infty .\end{cases}
$$

Solutions to Eqs. (4)-(6) are sought of the form

$$
\begin{aligned}
& \hat{\psi}_{m n}(r, t)=\hat{\psi}_{\mathrm{cs}}(r, t)+\hat{\psi}_{\mathrm{ew}}(r, t), \\
& \hat{q}_{m n}(r, t)=\hat{q}_{\mathrm{cs}}(r, t)+\hat{q}_{\mathrm{ew}}(r, t),
\end{aligned}
$$

where "cs" denotes the continuous spectrum and "ew" the Rossby edge-wave component. The solution method follows Smith and Montgomery (1995).

The continuous spectrum solution is given by

$$
\hat{\psi}_{\mathrm{cs}}(r, t)=\int_{0}^{\infty} G(r, \rho) \hat{q}_{0}(\rho) e^{-i n \bar{\Omega} t} \rho d \rho,
$$

where 


$$
G(r, \rho)=- \begin{cases}I_{n}\left(\gamma_{m} \rho\right) K_{n}\left(\gamma_{m} r\right), & \rho \geq r \\ I_{n}\left(\gamma_{m} r\right) K_{n}\left(\gamma_{m} \rho\right), & \rho \leq r\end{cases}
$$

is the Green function and $\hat{q}_{0}$ is the initial PV perturbation. $I_{n}$ and $K_{n}$ are the modified Bessel functions.

The edge-wave solution is found by solving the modified Bessel equation on both sides of the basic-state discontinuity. In both regions the mean vorticity gradient is identically zero. It follows that

$$
\hat{\psi}_{\mathrm{ew}}(r, t)=C_{n}(t) \hat{\phi}_{\mathrm{ew}}(r),
$$

where

$$
\hat{\phi}_{\mathrm{ew}}(r)=-a \begin{cases}I_{n}\left(\gamma_{m} r\right) K_{n}\left(\gamma_{m} a\right), & r \leq a \\ I_{n}\left(\gamma_{m} a\right) K_{n}\left(\gamma_{m} r\right), & r \geq a .\end{cases}
$$

The dynamic condition requires pressure continuity at $r=a$. From this condition $C_{n}(t)$ can be determined, completing the solution

$$
\begin{aligned}
C_{n}(t)= & -\frac{n \bar{\zeta}_{\max }}{a} \int_{0}^{\infty} \frac{G(a, \rho) \hat{q}_{0}(\rho)}{\sigma_{n}-n \bar{\Omega}} e^{-i n \bar{\Omega} t} \rho d \rho \\
& +\left\{C_{0}+\frac{n \bar{\zeta}_{\max }}{a} \int_{0}^{\infty} \frac{G(a, \rho) \hat{q}_{0}(\rho)}{\sigma_{n}-n \bar{\Omega} \rho} \rho d \rho\right\} e^{-i \sigma_{n} t},
\end{aligned}
$$

where $C_{0}$ is a constant of integration and

$$
\sigma_{n}=n \bar{\zeta}_{\max }\left[1 / 2-I_{n}\left(\gamma_{m} a\right) K_{n}\left(\gamma_{m} a\right)\right]
$$

is the edge-wave rotation frequency. A similar expression for $\sigma_{n}$ generalizable to mean vortices with multiple piecewise-continuous regions was derived by Waugh and Dritschel (1999).

This solution is intended to mimic the single-interface CD solution in the limit of infinitesimal vertical tilt. There are two points worth making regarding the linear solution. First, note that $\sigma_{n}$ is nonzero for all $n>0$ and $\gamma_{1}>0$. Therefore, as $t \rightarrow \infty$, oscillatory edge wave solutions exist for all wavenumbers. For continuous, relatively broad vortex monopoles, wavenumbers greater than 1 symmetrize within a couple $\tau_{e}$ (see Fig. 6). Second, note that as $\gamma_{1} \rightarrow \infty$ (Abramowitz and Stegun 1972),

$$
\begin{aligned}
K_{1} & \sim \sqrt{\frac{\pi}{2 a \gamma_{1}}} e^{-a \gamma_{1}} \text { and } \\
I_{1} & \sim \frac{1}{\sqrt{2 \pi a \gamma_{1}}} e^{a \gamma_{1}},
\end{aligned}
$$

so $\sigma_{1}$ approaches $\bar{\zeta}_{\max } / 2$ and wavenumber 1 propagates around the vortex indefinitely. This is consistent with the CD simulations of Polvani (1991) where corotation was found in this parameter regime. Continuous vortex monopoles do not exhibit this behavior except for the very steep-edged vortices discussed by Dritschel (1998) and Schecter et al. (2000).
As $\gamma_{1}$ increases, the Green function involved in the inversion of perturbation PV for streamfunction decays rapidly with radius. The convolution of perturbation PV and Green's function, which defines the streamfunction, will yield smaller values the larger $\gamma_{1}$ is. The radial advection of mean vorticity by the perturbation radial wind can then be neglected at zeroth order compared to the azimuthal advection of perturbation PV by the mean tangential wind (which does not depend on $\gamma_{1}$ ). The resulting linear PV tendency equation is

$$
\left(\frac{\partial}{\partial t}+i n \bar{\Omega}\right) \hat{q}_{m n}(r, t)=0
$$

Thus, the perturbation PV is materially conserved following the mean tangential winds and will take on a spiral pattern around the vortex in time since

$$
\hat{q}_{m n}(r, t)=\hat{q}_{m n}(r, 0) e^{-i n \Omega t} .
$$

The streamfunction is given by $\hat{\psi}_{\mathrm{cs}}$ above, and decays algebraically in the limit of long times. Therefore, continuous vortices always align in the small-tilt, large $\gamma_{1}$ limit.

\section{REFERENCES}

Abramowitz, M., and I. A. Stegun, 1972: Handbook of Mathematical Functions. Dover Publications, 1046 pp.

Bassom, A. P., and A. D. Gilbert, 1998: The spiral wind-up of vorticity in an inviscid planar vortex. J. Fluid Mech., 371, 109-140.

Bender, M. A., 1997: The effect of relative flow on the asymmetric structure in the interior of hurricanes. J. Atmos. Sci., 54, 703724.

Bister, M., and K. A. Emanuel, 1997: The genesis of Hurricane Guillermo: TEXMEX analyses and a modeling study. Mon. Wea. Rev., 125, 2662-2682.

Bracken, W. E., 1999: A multiscale examination of tropical cyclogenesis. Ph.D. dissertation, University at Albany, State University of New York, 340 pp. [Available from Dept. of Earth and Atmospheric Sciences, University at Albany, State University of New York, Albany, NY 12222.]

Carr, L. E., III, and R. T. Williams, 1989: Barotropic vortex stability to perturbations from axisymmetry. J. Atmos. Sci., 46, 31773191.

Case, K. M., 1960: Stability of inviscid plane Couette flow. Phys. Fluids, 3, 143-148.

DeMaria, M., 1996: The effect of vertical shear on tropical cyclone intensity change. J. Atmos. Sci., 53, 2076-2087.

Dritschel, D. G., 1998: On the persistence of non-axisymmetric vortices in inviscid two-dimensional flows. J. Fluid Mech., 371, $141-155$.

__ and M. de la Torre Juárez, 1996: The instability and breakdown of tall columnar vortices in a quasi-geostrophic fluid. J. Fluid Mech., 328, 129-160.

Emanuel, K. A., M. Fantini, and A. J. Thorpe, 1987: Baroclinic instability in an environment of small stability to slantwise moist convection. Part I: Two-dimensional models. J. Atmos. Sci., 44, 1559-1573.

Enagonio, J., and M. T. Montgomery, 2001: Tropical cyclogenesis via convectively forced vortex Rossby waves in a shallow water primitive equation model. J. Atmos. Sci., 58, 685-705.

Farge, M., and R. Sadourny, 1989: Wave-vortex dynamics in rotating shallow water. J. Fluid Mech., 206, 433-462.

Frank, N. L., 1975: Atlantic tropical systems of 1974. Mon. Wea. Rev., 103, 294-300. 
Frank, W. M., and E. A. Ritchie, 1999: Effects of environmental flow upon tropical cyclone structure. Mon. Wea. Rev., 127, 20442061.

Gent, P. R., and J. C. McWilliams, 1986: The instability of barotropic circular vortices. Geophys. Astrophys. Fluid Dyn., 35, 209-233.

Gray, W. M., 1968: Global view of the origin of tropical disturbances and storms. Mon. Wea. Rev., 96, 669-700.

Hawkins, H. F., and D. T. Rubsam, 1968: Hurricane Hilda, 1964. II. The structure and budgets of the hurricane on October 1, 1964. Mon. Wea. Rev., 96, 617-636.

Held, I. M., and P. J. Phillips, 1987: Linear and nonlinear barotropic decay on the sphere. J. Atmos. Sci., 44, 200-207.

Holton, J. R., 1992: An Introduction to Dynamic Meteorology. 3d ed. Academic Press, 511 pp.

Hoskins, B. J., and F. P. Bretherton, 1972 Atmospheric frontogenesis models: Mathematical formulation and solution. J. Atmos. Sci., 29, 11-37.

— M. E. McIntyre, and A. W. Robertson, 1985: On the use and significance of isentropic potential vorticity maps. Quart. J. Roy. Meteor. Soc., 111, 877-946.

Jones, S. C., 1995: The evolution of vortices in vertical shear: Initially barotropic vortices. Quart. J. Roy. Meteor. Soc., 121, 821-851.

Marks, F. D., R. A. Houze, and J. Gamache, 1992: Dual-aircraft investigation of the inner core of Hurricane Norbert. Part 1: Kinematic structure. J. Atmos. Sci., 49, 919-942.

McWilliams, J. C., 1989: Statistical properties of decaying geostrophic turbulence. J. Fluid Mech., 198, 199-230.

__ , and G. R. Flierl, 1979: On the evolution of isolated, nonlinear vortices. J. Phys. Oceanogr., 9, 1155-1182.

Melander, M. V., J. C. McWilliams, and N. J. Zabusky, 1987: Axisymmetrization and vorticity-gradient intensification of an isolated two-dimensional vortex through filamentation. J. Fluid Mech., 178, 137-159.

Michalke, A., and A. Timme, 1967: On the inviscid instability of certain two-dimensional vortex-type flows. J. Fluid Mech., 29, 647-666.

Möller, J. D., and M. T. Montgomery, 1999: Vortex Rossby waves and hurricane intensification in a barotropic model. J. Atmos. Sci., 56, 1674-1687.

_ ticity anomalies in a three-dimensional balance model. J. Atmos. Sci., 57, 3366-3387.

Montgomery, M. T., and B. F. Farrell, 1992: Polar low dynamics. J. Atmos. Sci., 49, 2484-2505.

—_ and R. Kallenbach, 1997: A theory for vortex Rossby-waves and its application to spiral bands and intensity changes in hurricanes. Quart. J. Roy. Meteor. Soc., 123, 435-465.

_ forced vortex Rossby waves in a three-dimensional quasigeostrophic model. J. Atmos. Sci., 55, 3176-3207.

_ J. D. Möller, and C. T. Nicklas, 1999: Linear and nonlinear vortex motion in an asymmetric balance shallow water model. J. Atmos. Sci., 56, 749-768.

- J. M. Hidalgo, and P. D. Reasor, 2000: A semi-spectral numerical model for modeling the vorticity dynamics of the nearcore region of hurricane-like vortices. Atmospheric Science Paper 695, Colorado State University, 56 pp. [Available from Dept. of Atmospheric Science, Colorado State University, Fort Collins, CO 80523.]

Polvani, L. M., 1991: Two-layer geostrophic vortex dynamics. Part 2. Alignment and two-layer V-states. J. Fluid Mech., 225, 241270 .

Reasor, P. D., 2000: Horizontal vorticity redistribution and vortex alignment in developing and mature tropical cyclones. Ph.D. dissertation, Colorado State University, 166 pp. [Available from Dept. of Atmospheric Science, Colorado State University, Fort Collins, CO 80523.]

— M. T. Montgomery, F. D. Marks Jr., and J. F. Gamache, 2000: Low-wavenumber structure and evolution of the hurricane inner core observed by airborne dual-Doppler radar. Mon. Wea. Rev., 128, 1653-1680.

Rivest, C., and B. F. Farrell, 1992: Upper-tropospheric synoptic-scale waves. Part II: Maintenance and excitation of quasi modes. $J$. Atmos. Sci., 49, 2120-2138.

Schecter, D. A., D. H. E. Dubin, A. C. Cass, C. F. Driscoll, I. M. Lansky, and T. M. O'Neil, 2000: Inviscid damping of asymmetries on a two-dimensional vortex. Phys. Fluids, 12, 2397 2412.

Shapiro, L. J., and M. T. Montgomery, 1993: A three-dimensional balance theory for rapidly rotating vortices. J. Atmos. Sci., 50, 3322-3335

Smith, G. B., and M. T. Montgomery, 1995: Vortex axisymmetrization: Dependence on azimuthal wavenumber or asymmetric radial structure changes. Quart. J. Roy. Meteor. Soc., 121, 16151650 .

Smith, R. K., W. Ulrich, and G. Sneddon, 2000: On the dynamics of hurricane-like vortices in vertical shear flows. Quart. J. Roy. Meteor. Soc., 126, 2653-2670.

Sutyrin, G. G., 1989: Azimuthal waves and symmetrization of an intense vortex. Sov. Phys. Dokl., 34, 104-106.

_ , J. C. McWilliams, and R. Saravanan, 1998: Co-rotating stationary states and vertical alignment of geostrophic vortices with thin cores. J. Fluid Mech., 357, 321-349.

Viera, F., 1995: On the alignment and axisymmetrization of a vertically-tilted geostrophic vortex. J. Fluid. Mech., 289, 29-50.

Waugh, D. W. and D. G. Dritschel, 1999: The dependence of Rossby wave breaking on the vertical structure of the polar vortex. $J$. Atmos. Sci., 56, 2359-2375.

Weber, H. C., and R. K. Smith, 1993: The stability of barotropic vortices: Implications for tropical cyclone motion. Geophys. Astrophys. Fluid Dyn., 70, 1-30.

Willoughby, H. E., 1990: Temporal changes of the primary circulation in tropical cyclones. J. Atmos. Sci., 47, 242-264.

Zehr, R., 1992: Tropical cyclogenesis in the Western North Pacific. NOAA Tech. Rep. NESDIS 61, 181 pp. 